\title{
General-purpose neural network interatomic potential for the $\alpha$-iron and hydrogen binary system: Toward atomic-scale understanding of hydrogen embrittlement
}

\author{
Fan-Shun Meng $\odot,{ }^{1,2, *}$ Jun-Ping Du $\odot,{ }^{3,1}$ Shuhei Shinzato $\odot,{ }^{1}$ Hideki Mori $\odot,{ }^{4}$ Peijun Yu $\odot,{ }^{1}$ Kazuki Matsubara, ${ }^{5}$ \\ Nobuyuki Ishikawa, ${ }^{5}$ and Shigenobu Ogata $\oplus^{1,3, \dagger}$ \\ ${ }^{1}$ Department of Mechanical Science and Bioengineering, Graduate School of Engineering Science, Osaka University, \\ 1-3 Machikaneyama, Toyonaka, Osaka 560-8531, Japan \\ ${ }^{2}$ School of Science, Liaoning University of Technology, Jinzhou 121001, China \\ ${ }^{3}$ Center for the Element Strategy Initiative for Structural Materials (ESISM), Kyoto University, Sakyo-ku, Kyoto 606-8501, Japan \\ ${ }^{4}$ Department of Mechanical Engineering, College of Industrial Technology, Hyogo 661-0047, Japan \\ ${ }^{5}$ Steel Research Laboratory, JFE Steel Corporation, Kawasaki 210-0855, Japan
}

(Received 16 August 2021; revised 7 October 2021; accepted 26 October 2021; published 22 November 2021)

\begin{abstract}
To understand the physics of hydrogen embrittlement at the atomic scale, a general-purpose neural network interatomic potential (NNIP) for the $\alpha$-iron and hydrogen binary system is presented. It is trained using an extensive reference database produced by density functional theory (DFT) calculations. The NNIP can properly describe the interactions of hydrogen with various defects in $\alpha$-iron, such as vacancies, surfaces, grain boundaries, and dislocations; in addition to the basic properties of $\alpha$-iron itself, the NNIP also handles the defect properties in $\alpha$-iron, hydrogen behavior in $\alpha$-iron, and hydrogen-hydrogen interactions in $\alpha$-iron and in vacuum, including the hydrogen molecule formation and dissociation at the $\alpha$-iron surface. These are superb challenges for the existing empirical interatomic potentials, like the embedded-atom method based potentials, for the $\alpha$-iron and hydrogen binary system. In this study, the NNIP was applied to several key phenomena necessary for understanding hydrogen embrittlement, such as hydrogen charging and discharging to $\alpha$-iron, hydrogen transportation in defective $\alpha$-iron, hydrogen trapping and desorption at the defects, and hydrogen-assisted cracking at the grain boundary. Unlike the existing interatomic potentials, the NNIP simulations quantitatively described the atomistic details of hydrogen behavior in the defective $\alpha$-iron system with DFT accuracy.
\end{abstract}

DOI: 10.1103/PhysRevMaterials.5.113606

\section{INTRODUCTION}

The hydrogen-induced mechanical performance degradation of metals and all phenomena associated with its deleterious effects are known as hydrogen embrittlement (HE) $[1,2]$, which is a prominent problem in current societal and technological requirements [3]. Iron and its alloys are the most widely used structural materials in various industries due to their excellent mechanical properties and low cost. The development of iron alloys with HE resistance requires a deep understanding of the interaction between hydrogen and the defects in the iron alloy's matrix [4]. Experimental examination of the role played by hydrogen in metals is extremely challenging owing to its high diffusivity, low solubility, and small size [5]. As an alternative solution, computer simulations at the atomic level can provide insightful information. Electronic structure calculations, such as density functional theory (DFT), supply the most accurate potential

\footnotetext{
*Author to whom correspondence should be addressed: fanshun.meng@tsme.me.es.osaka-u.ac.jp

†ogata@me.es.osaka-u.ac.jp

Published by the American Physical Society under the terms of the Creative Commons Attribution 4.0 International license. Further distribution of this work must maintain attribution to the author $(s)$ and the published article's title, journal citation, and DOI.
}

energy surfaces (PESs). However, the high computational cost limits their application to large-scale simulations despite the remarkable progress made in computational power and algorithms. Unlike DFT calculations, the molecular dynamics (MD) method with empirical interatomic potentials (EIPs) can simulate large systems at long time scales, but they strongly rely on the quality of EIPs; the accuracy and transferability of EIPs are always questionable [6].

Several EIPs, including the embedded atom method (EAM) and modified EAM (MEAM) potentials, for $\mathrm{H}$ atoms in $\alpha$-iron have been proposed [7-10] and have made remarkable contributions to the understanding of HE. Among these EIPs, two EAM potentials for the Fe-H system [10] based on iron potentials, proposed by Mendelev et al. [11] and Ackland et al. [12], can produce the corrected atomic structure of screw dislocation. Unfortunately, they cause strong attractive interaction among $\mathrm{H}$ atoms, causing $\mathrm{H}$ atoms to cluster in the matrix at moderate temperatures. Although these potentials have been improved by introducing an additional repulsive term for $\mathrm{H}-\mathrm{H}$ interactions [13] or by refitting the parameters of the $\mathrm{Fe}-\mathrm{H}$ and $\mathrm{H}-\mathrm{H}$ interactions [14], the problem remains in scenarios with high hydrogen concentration. Another severe drawback of such potentials is their poor description of the energetics of screw dislocation $[15,16]$. More information on the existing $\mathrm{Fe}-\mathrm{H}$ EIPs is provided in the latest review paper on this topic [17].

Consequently, an interatomic potential for the $\alpha$-iron and hydrogen binary system that combines the advantages of 
the accuracy of DFT calculations and computational cost of EIPs needs to be constructed. Advances in machine learning methods allow the attainment of this goal. In principle, machine-learning-based interatomic potentials (MLBIPs) can approximate any function with arbitrary accuracy [16]. MLBIPs, such as neural network interatomic potentials (NNIPs) [18] and Gaussian approximation potentials (GAPs) [19], have been proposed and successfully applied to many systems [20-23]. Recently, Davidson et al. [24] proposed a purposemade $\mathrm{H}-\mathrm{Fe}$ Gaussian approximation potential that enables rapid sampling of $\mathrm{H}$ atoms in a vacancy with DFT-level accuracy, and reported novel results, while the potential does not contain the $\mathrm{Fe}-\mathrm{Fe}$ interaction. Although this is a wise strategy for constructing a purpose-made MLBIP that solves a specific target problem, a target involving multiple defects requires a general-purpose MLBIP.

In this study, the NNIP framework was adopted and a general-purpose NNIP for the $\alpha$-iron and hydrogen binary system was constructed. The constructed NNIP can well reproduce the interactions of hydrogen with various defects in $\alpha$-iron, such as vacancies, surfaces, grain boundaries, and dislocations; in addition to the basic properties of $\alpha$-iron itself, the NNIP can also handle the defect properties, hydrogen behavior, and hydrogen-hydrogen interactions in $\alpha$-iron and in vacuum, including the hydrogen molecule formation and dissociation at the $\alpha$-iron surface. These properties are essential for understanding the physics of $\mathrm{HE}$, which cannot be fully described by the existing interatomic potentials. Eventually, the constructed general-purpose NNIP was applied to several key phenomena underlying $\mathrm{HE}$ in $\alpha$-iron with point defect, dislocation, surface, and grain boundaries. These phenomena include hydrogen charging and discharging, hydrogen transportation, hydrogen trapping and desorption at defects, and hydrogen-assisted cracking at the grain boundary. The simulations uncovered the atomistic details of these phenomena in DFT accuracy.

\section{METHODOLOGY}

\section{A. Reference database and neural network architecture}

To learn and reproduce is the essence of MLBIPs [25,26]. The NNIP quality relies on the reference database, and NNIP transferability depends to a certain extent on the similarity of structures presented in actual simulations against those in the reference database. A broad spectrum of structures but limited to important structures that directly related the target properties were considered. In total, 21928 configurations, equivalent to $1.9 \times 10^{6}$ atomic environments, were prepared in the reference database (see Sec. SI of the Supplemental Material [27] for details). Both the total energy and three force components of each atom were included for each structure [28]. The structures in the reference database were randomly distributed into training data set $(90 \%)$ and testing data set (10\%), which were used to fit the adjustable parameters and check the quality of the potential for unlearned structures. The neural network (NN) architecture has two hidden layers, each of which possesses 15 neurons (see Fig. S2 in the Supplemental Material [27]). The activation function is set as a logistic form in both hidden layers. Sixteen radial and
48 angular atom-centered symmetry functions (ACSFs) [29] were adopted for each element (see Sec. II B for details of the ACSF settings); 1231 adjustable parameters (i.e., 1200 weights and 31 biases) were used for each element. The entire NNIP training task was completed using the neural network potential package $(n 2 p 2)$ [30]. The constructed NNIP can be applied in the LAMMPS code [31] via the interface implemented in the $n 2 p 2$ package [32], and both the reference database and potential files are shared online [33].

\section{B. Calculation settings}

ACSFs settings. Two types of ACSFs [29], namely, radial symmetry functions and angular symmetry functions, were adopted to distinguish the local atomic environments within the cutoff radius. The radial symmetry function is defined as

$$
G_{i}^{\mathrm{rad}}=\sum_{j} e^{-\eta\left(R_{i j}-R_{s}\right)^{2}} f_{c}\left(R_{i j}\right),
$$

where $R_{i j}$ is the atomic distance between an atom $j$ and the central atom $i$. The angular symmetry function is given by

$$
G_{i}^{\text {ang }}=2^{1-\xi} \sum_{j} \sum_{k \neq j}\left(1+\lambda \cos \theta_{i j k}\right)^{\xi} e^{-\eta\left(R_{i j}+R_{i k}\right)^{2}} f_{c}\left(R_{i j}\right) f_{c}\left(R_{i k}\right),
$$

where $\theta_{i j k}$ is the angle enclosed by the vectors of $R_{i j}$ and $R_{i k}$ of two neighboring atoms $j$ and $k$, respectively. Both types of ACSF have a common function $f_{c}$ called the cutoff function, which is defined as follows:

$$
f_{c}\left(R_{i j}\right)= \begin{cases}\tanh ^{3}\left[1-\frac{R_{i j}}{R_{c}}\right] & \text { if } R_{i j} \leqslant R_{c} \\ 0.0 & \text { if } R_{i j}>R_{c} .\end{cases}
$$

Here, $R_{c}$ is the cutoff radius. The parameters $\eta, R_{s}, \xi$, and $\lambda$ in Eqs. (1) and (2) can be determined by the strategy reported in Ref. [34]. The cutoff radii are 6.5 and $3.6 \AA$ for $F e$ and $H$ atoms, respectively.

DFT calculation settings. The reference database was produced by spin-polarized DFT calculations using the VASP code [35]. The electron-ion interaction was described using the projector augmented wave [36] method. The exchangecorrelation energy was treated with the GGA-PBE approach [37]. The Brillouin zone (BZ) was sampled using the Monkhorst-Pack scheme, and the BZ integration employed the Methfessel-Paxton method for relaxations. The smallest allowed spacing between $k$-points was $0.03 \AA^{-1}$, and the energy smearing was $0.1 \mathrm{eV}$. A cutoff energy of $360 \mathrm{eV}$ was employed to truncate the plane-wave expansion of the wave functions. Geometry optimizations were continued until the force in the system was less than $10^{-2} \mathrm{eV} / \AA$. The stopping criterion for the electronic structure optimization was set to $10^{-5} \mathrm{eV}$. To enlarge the coverage area of the PES, the reference database was expanded by $a b$ initio MD (AIMD) sampling. AIMD was performed in the canonical ensemble with a Nosé-Hoover thermostat implemented in the VASP code. All systems were simulated within 100-1000 K (up to $2000 \mathrm{~K}$ in liquid configurations only) with a time step of 1-2 fs. The transition state between the initial and final atomic configurations was calculated using the climbing image nudge elastic band (CI-NEB) method. The zero-point vibration energy was not included, but if needed, can be incorporated (for 


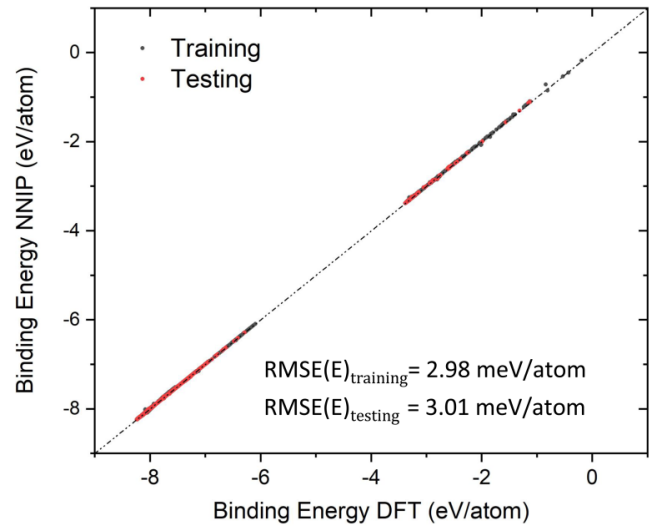

(a)

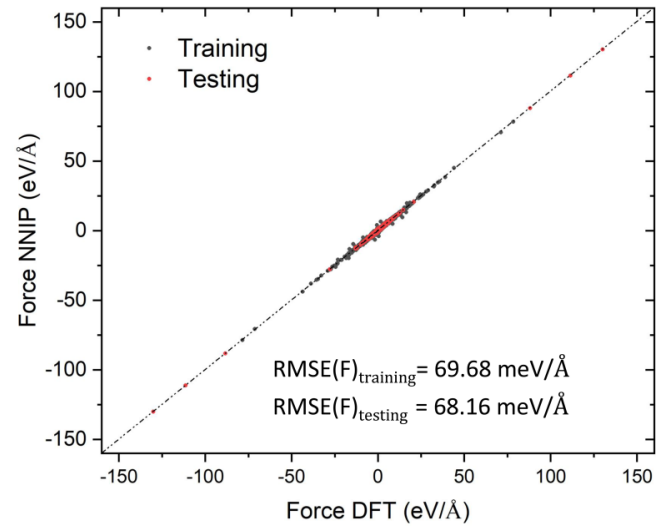

(b)

FIG. 1. Comparison of DFT and NNIP (a) energies and (b) forces of the structures in the training and testing data sets. The line with a slope of 1 corresponds to a perfect training.

example) by the path-integral method [38] when using the NNIP in a simulation.

\section{RESULTS: VALIDATION}

\section{A. Overall NNIP accuracy}

Owing to the possibility of trapping in local minima in the NN learning process, many NNIP trial trainings should be performed with different initial states, and many candidates should be obtained. Among these candidates we should choose the best $\mathrm{NN}$ that well describes not only the training data set but also the testing data set. The NNIP quality is estimated by the root-mean-squared error (RMSE) of energies $(E)$ and forces $(F)$ primarily used to guide the optimization of adjustable parameters:

$$
\begin{gathered}
\operatorname{RMSE}(E)=\sqrt{\frac{1}{N_{\mathrm{s}}} \sum_{i}^{N_{\mathrm{s}}}\left(E_{\mathrm{NNIP}}^{i}-E_{\mathrm{DFT}}^{i}\right)^{2},} \\
\operatorname{RMSE}(F)=\sqrt{\frac{1}{N_{\mathrm{s}}} \sum_{i}^{N_{\mathrm{s}}} \frac{1}{3 N_{\mathrm{a}}} \sum_{\alpha}^{3 N_{\mathrm{a}}}\left(F_{\alpha, \mathrm{NNIP}}^{i}-F_{\alpha, \mathrm{DFT}}^{i}\right)^{2},}
\end{gathered}
$$

where $N_{\mathrm{s}}$ and $N_{\mathrm{a}}$ denote the number of structures in the training and testing data sets and the number of atoms in individual structures, respectively. The NNIP with the highest quality among all candidates has a low RMSE and good reproducibility and transferability of the important properties; this was adopted and addressed in detail in the following text.

As shown in Fig. 1, the training and testing data sets have $\operatorname{RMSE}(E) \mathrm{s}$ of 2.98 and $3.01 \mathrm{meV} /$ atom, and $\operatorname{RMSE}(F) \mathrm{s}$ of 69.68 and $68.16 \mathrm{meV} / \AA$, respectively. These RMSEs are sufficiently small because they approach the usual accuracy of DFT analysis, and the small difference between the RMSEs of training and testing data sets denotes that no serious overfitting occurred [39]. Just in case, an additional data set including 3240 configurations, produced by AIMD simulations at higher temperature for the different systems, was prepared and further confirmed the no-overfitting issue (see Sec. SIII of the Supplemental Material [27]). All points for energy and force are very close to the line with a slope of 1 , implying that the trained data can be well reproduced. Several concerned properties are further confirmed in the following sections. These properties following the data source categories are fully included, partially included, and not included, and also of the test results are tabulated in Table S3 in the Supplemental Material [27].

\section{B. NNIP performance for pure $\alpha$-iron}

The NNIP of the Fe-H binary system must be reliable also for $\alpha$-iron. The basic properties of iron, such as lattice constant [40-43], elastic constants [44], vacancy cluster formation energies [45-47], self-interstitial formation energies [48,49], low index surface energies [50], Bain path, and energy-volume relations [16] are summarized in Table S4 and Fig. S4 in the Supplemental Material [27]. All of the above-mentioned properties are in good agreement with the DFT results, and most of them are included in the reference database (see Sec. SIV of the Supplemental Material [27]).

Phonon dispersions of $\alpha$-iron under an equilibrium lattice condition with the supercell size of $3 \times 3 \times 3$ were computed using the PHONOPY code [55] and displayed in Fig. 2(a). The results produced by NNIP show an excellent agreement with DFT [16,50] and with the experimental [51] results. The training data set contains the energy and force information of the AIMD snapshots at finite temperatures, which is sufficient to obtain the phonon and vibration properties of iron.

$\gamma$ surfaces, or generalized stacking fault (GSF) energies [56], of the (110) and (112) planes (the dominant slip planes in bcc metals [57]) were considered. The $\gamma$ surface was calculated by in-plane shifting between upper and lower halves in the supercell. In-plane atomic motions were fixed while those in the normal direction were allowed. The results are displayed in Fig. 2(b) and Fig. S4(f) in the Supplemental Material [27]. We confirmed that the NNIP can reproduce the reported $\gamma$ surface [50]. Note that as mentioned above, the $\gamma$ surface energies are included in the reference database.

Grain boundaries (GBs) are another important defect for the polycrystalline plasticity and fracture. Therefore, it is strongly expected that the NNIP can describe various GB structures and energetics. If the NNIP can well describe the 


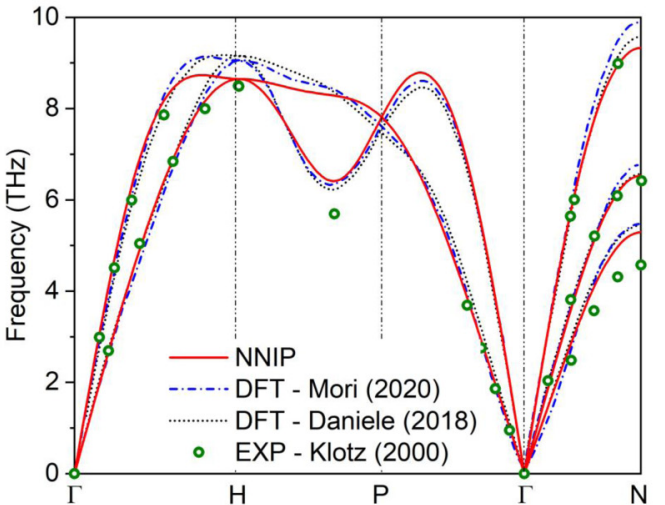

(a)

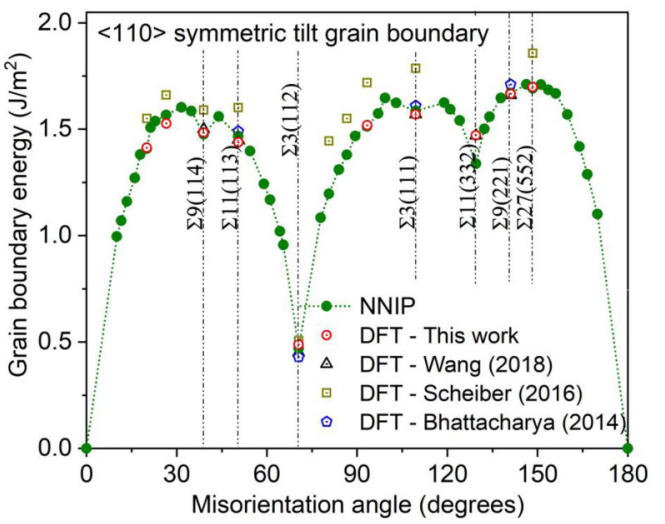

(c)

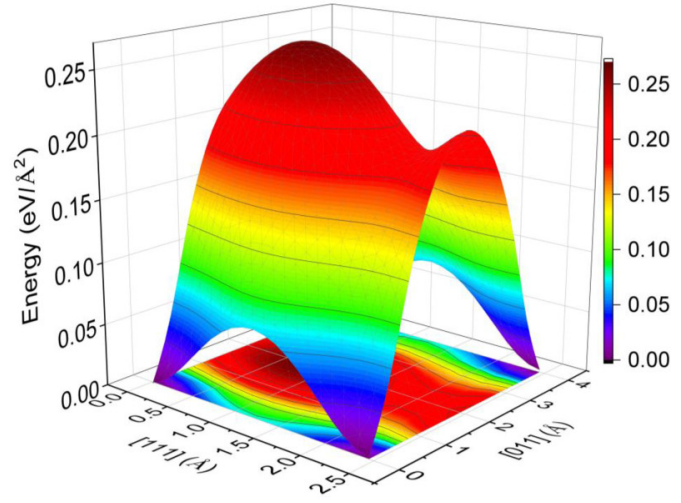

(b)

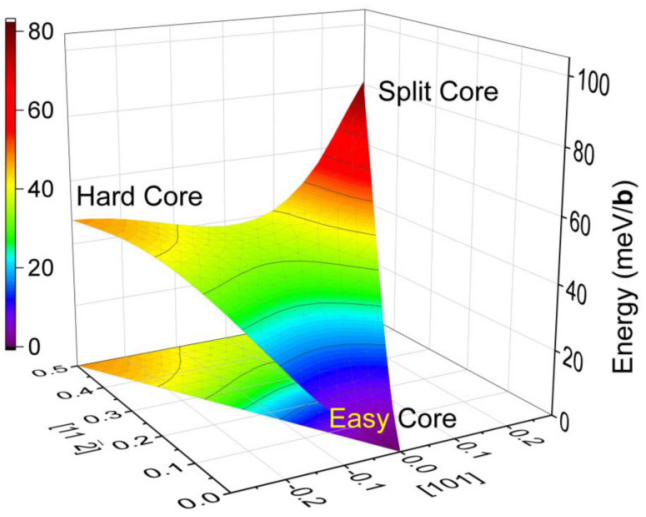

(d)

FIG. 2. NNIP performance for $\alpha$-iron. (a) Phonon dispersion curve, (b) $\gamma$ surface for the (112) crystallographic orientation, (c) misorientation-energy relationship for the symmetric tilt GB with $\langle 110\rangle$ tilt axis, and (d) 2D Peierls potentials. The axes along [101] and [112] directions in (d) are scaled by $\sqrt{3} a_{0} / 6 ; a_{0}$ is the lattice constant of $\alpha$-iron. The available reported DFT [16,50] and experimental [51] results for phonon dispersion curves and DFT results for symmetric tilt GB formation energy [52-54] are also shown.

GB structure and energetics, it should also well describe edge dislocations because low-angle GBs are constituted by an array structure of edge dislocations. We mapped the misorientation versus the formation energy of symmetric GBs with tilt axes of $\langle 110\rangle,\langle 001\rangle$, and $\langle 111\rangle$. The result for the $\langle 110\rangle$ tilt axis is plotted in Fig. 2(c). As marked by red circles in Fig. 2(c), $10 \mathrm{GBs}$ with misorientation in the range of $20^{\circ}$ to $148^{\circ}$ are included in the reference database. Compared to the DFT results, NNIP accurately reproduces the formation energies of the trained GBs, and, more importantly, it correctly predicts the formation energies of other GBs [52-54], which are not included in the reference database. The maps for GBs with tilt axes of $\langle 001\rangle$ and $\langle 111\rangle$ also show good agreement with DFT results (see Figs. S4(d) and S4(e) in the Supplemental Material [27]).

Screw dislocation motion is essential for the plasticity of bcc metals [58], and the core structure of screw dislocation and motion of kink pairs is believed to be attributed to that behavior [59-61]. To examine the core structure of screw dislocation, we used a supercell with dimensions of 137.4, 2.45 , and $40.3 \AA$ in the [112̄], [111], and [11̄0] directions, respectively, comprising a total of $1200 \mathrm{Fe}$ atoms. Periodic boundaries are taken along the [112]] and [111] directions. A $\frac{1}{2}$ [111] screw dislocation with easy core configuration is embedded at the center of the simulation cell. To study the dislocation motion, a large model, that is 40 times larger than the 1200-Fe-atom model in the [111] direction, was constructed (see Fig. S5(a) in the Supplemental Material [27]). This periodic array of dislocation (PAD) configuration is widely used in atomic simulations [62,63]. Our NNIP can correctly predict the atomic structures of easy core, hard core, and split core (differential displacement maps for the three core configurations are shown in Figs. S5(b)-S5(d) in the Supplemental Material [27]) and can also satisfactorily predict their energetics. The two-dimensional (2D) Peierls potential [Fig. 2(d)] was obtained using numerical interpolation from the corresponding energy profiles along the pathways among the above-mentioned atomic configurations; in total, 28 configurations were adopted. The 2D Peierls potential vividly matches that reported in Refs. [15,64], although only the data on the straight paths from one easy core state to a neighboring easy core state and from hard core to split core states are included in the reference database using a small 150-Fe-atom model with various screw dislocation core configurations at center (see Fig. S1(a) in the Supplemental Material [27]). Clearly, the Peierls barrier between the two neighboring easy core configurations has only one hump, which has important consequences on the kink pair formation mechanism [65] and most of the EAM potentials cannot reproduce the onehump shape [50]. Compared to the energy of the easy core 


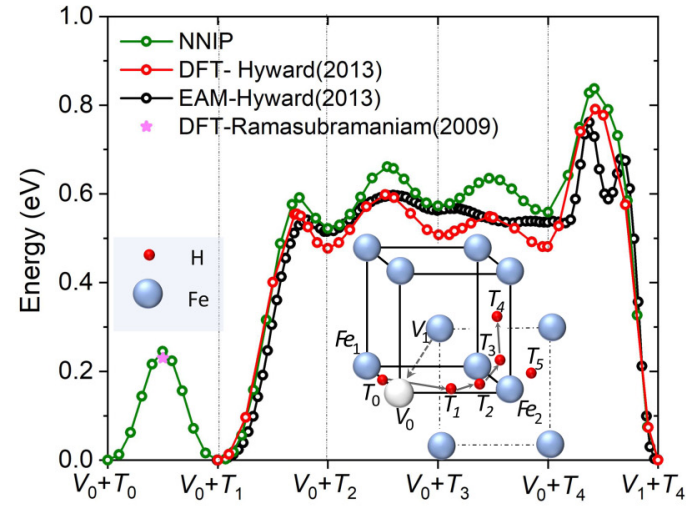

(a)

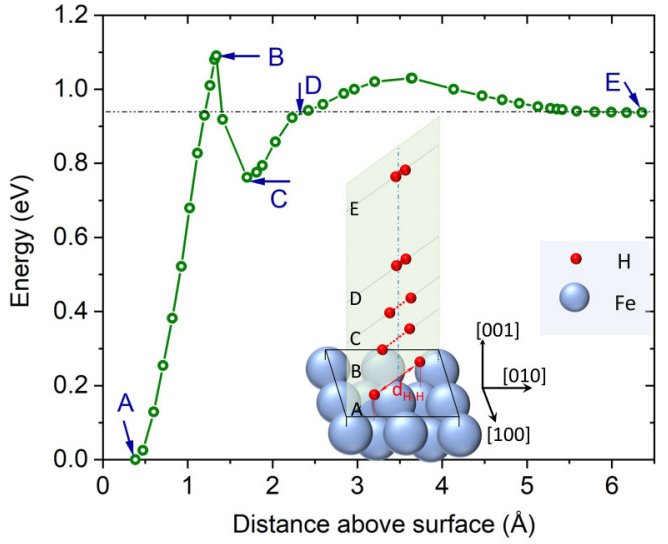

(b)

FIG. 3. (a) Migration barriers of the hydrogen-vacancy complex. DFT and EAM results extracted from Ref. [10] and Ref. [81] are also plotted. Locations of vacancy and $\mathrm{H}$ atom indicated by $\mathrm{V}_{m}+\mathrm{T}_{n}(m=0-1$ and $n=0-5)$, where $\mathrm{V}_{m}$ and $\mathrm{T}_{n}$ indicate the location of vacancy and $\mathrm{H}$ atom, respectively, and the schematic diagram is presented in the inset of (a). (b) Hydrogen dissociation on the (001) surface. Positions of hydrogen atoms in several interesting locations are depicted in the inset of (b).

configuration, the energies of the configurations of the hard core, split core, and the middle point, respectively, are 47.4, 82.3, and $38.2 \mathrm{meV} / b$, which reasonably agree with DFT results of $39.3,108$, and $37.9 \mathrm{meV} / b$ [15], and 33.2, 87.9, and $34.9 \mathrm{meV} / b$ [64], and 57.9, 110.3 , and $49.2 \mathrm{meV} / b$ [66], where $b$ is the Burgers vector length, i.e., $\sqrt{3} a_{0} / 2\left(a_{0}\right.$ is the lattice constant of $\alpha$-iron). Screw dislocation gliding and kink pair nucleation under shear stress were further tested (see Sec. IV of the Supplemental Material [27]).

\section{Hydrogen solubility and diffusivity in bulk $\alpha$-iron}

The solution energy and diffusion barrier of the $\mathrm{H}$ atom should be reproduced to successfully demonstrate the hydrogen charging and diffusion phenomena. In $\alpha$-iron, $\mathrm{H}$ atoms preferentially settle at two interstitial sites: the tetrahedral (T) and octahedral $(\mathrm{O})$ sites. The solution energy of the $\mathrm{H}$ atom in bulk, $E_{\mathrm{s}}$, is calculated as follows:

$$
E_{\mathrm{s}}=E_{\mathrm{Fe}+\mathrm{H}}-E_{\mathrm{Fe}}-E_{\mathrm{H}_{2}} / 2,
$$

where $E_{\mathrm{Fe}+\mathrm{H}}$ and $E_{\mathrm{Fe}}$ are the energies of Fe bulk with and without $\mathrm{H}$ atom at the interstitial site, respectively, and $E_{\mathrm{H}_{2}}$ is the energy of a gaseous $\mathrm{H}_{2}$ molecule. The $E_{\mathrm{s}}$ of a $\mathrm{H}$ atom at the $\mathrm{T}$ site obtained by NNIP is $0.236 \mathrm{eV}$, which reasonably agrees with the DFT results of 0.22 [67], 0.234 [68], and $0.21 \mathrm{eV}$ [69]. The $E_{\mathrm{s}}$ for the $\mathrm{H}$ atom at the $\mathrm{O}$ site is determined as $0.389 \mathrm{eV}$ using NNIP, which matches the reported DFT result of $0.35 \mathrm{eV}$ [70]. The diffusion energy barrier, $\Delta E$, for $\mathrm{H}$-atom diffusion between neighboring $\mathrm{T}$ sites predicted by NNIP is $0.108 \mathrm{eV}$, which is in line with our DFT result of $0.092 \mathrm{eV}$ as well as other DFT and experimental results of 0.088 [71], 0.096 [72], and 0.035-0.14 eV [53]. The distance from the saddle point in the T-T diffusion path to the nearest $\mathrm{O}$ site is $0.368 \AA$, which is consistent with a previously reported DFT result of $0.407 \AA$ [71]. All of the above data are included in the reference database. The diffusivity of $\mathrm{H}$ atoms in $\alpha$-iron bulk [73-75] and the volumetric engineering strain dependent hydrogen solution energy and diffusion barrier were further tested and provided in the Supplemental Material (Secs. SV and SVI) [27].

\section{Hydrogen-vacancy interaction}

Hydrogen trapping by defects is an essential phenomenon in hydrogen embrittlement of structural metallic metals [76] because it leads to an extremely high localized $\mathrm{H}$ concentration [77], then leads to the defects growth, such as void or bubble formation [67] and crack initiation and propagation. First, we systematically confirmed the interaction between monovacancy and various number of $\mathrm{H}$ atoms, which demonstrated fairly good agreement with the reference database and DFT results in the literature [78-81]. The details of the trapping configurations and trapping energies can be found in the Supplemental Material (Sec. SVII) [27].

Next, we paid more attention to the diffusion of the $\mathrm{H}$ vacancy complex because it is still challenging for empirical potentials [81]. The energy profile of the H-vacancy complex in the diffusion pathway outlined in Ref. [81] is predicted using the CI-NEB method with NNIP, as shown in Fig. 3(a). The diffusion barrier for the H-vacancy complex $\left(\mathrm{V}_{0}+\mathrm{T}_{1} \rightarrow\right.$ $\mathrm{V}_{1}+\mathrm{T}_{4}$ ) is $0.837 \mathrm{eV}$, which is higher than that of a $\mathrm{H}$-free monovacancy of $0.73 \mathrm{eV}$. The corresponding DFT results are 0.79 and $0.69 \mathrm{eV}$, respectively [81]. The EAM potential can reasonably predict the diffusion barrier, $0.78 \mathrm{eV}$; however, the details of the energy profile differ from the DFT result [81], especially the double hump presented at the final vacancy jump $\left(\mathrm{V}_{0}+\mathrm{T}_{4} \rightarrow \mathrm{V}_{1}+\mathrm{T}_{4}\right)$. In addition, the barrier that the $\mathrm{H}$ atom has to overcome to jump from vacancy to a neighboring $\mathrm{T}$ site $\left(\mathrm{V}_{1}+\mathrm{T}_{3} \rightarrow \mathrm{V}_{1}+\mathrm{T}_{5}\right)$ is $0.591 \mathrm{eV}$; this is very close to the DFT results of 0.554 [81], 0.583 [72], and $0.650 \mathrm{eV}$ [10]. The barrier for the $\mathrm{H}$ atom to jump into a vacancy from a neighboring $\mathrm{T}$ site $\left(\mathrm{V}_{1}+\mathrm{T}_{5} \rightarrow \mathrm{V}_{1}+\mathrm{T}_{3}\right)$ is $0.069 \mathrm{eV}$; this is also in agreement with the DFT results of 0.078 [81], 0.09 [72], and $0.033 \mathrm{eV}$ [10]. Note that these energy barriers are included in the reference database.

More importantly, it is difficult for the EAM potentials to predict the diffusion barrier between trapping sites in a 
vacancy [10], such as $\mathrm{V}_{0}+\mathrm{T}_{0} \rightarrow \mathrm{V}_{0}+\mathrm{T}_{1}$ [inset of Fig. 3(a)]. By virtue of the flexibility of NNIP, the above-mentioned energy barrier predicted by NNIP is $0.245 \mathrm{eV}$, which is in close agreement with the DFT result of $0.230 \mathrm{eV}$, whereas the EAM result is $0.061 \mathrm{eV}$ [10]. Interestingly, the energy barrier of $0.245 \mathrm{eV}$ is significantly larger than that recorded in bulk of $0.108 \mathrm{eV}$, even when the free volume in vacancy is larger than that in bulk. This could be explained by the fact that the $\mathrm{H}$ atom at $\mathrm{T}_{0}$ (abbreviated as $\mathrm{H} @ \mathrm{~T}_{0}$ ) bonded with its neighboring $\mathrm{Fe}_{1}$ atom through a covalent bond of $\mathrm{Fe} 3 d-\mathrm{H} 1 s$ [78]. To accomplish a jump from $\mathrm{T}_{0}$ to $\mathrm{T}_{1}$, the $\mathrm{Fe}_{1}-\mathrm{H} @ \mathrm{~T}_{0}$ covalent bond must be broken, for which a large energy barrier needs to be conquered before forming the $\mathrm{Fe}_{2}-\mathrm{H} @ \mathrm{~T}_{1}$ bond. This suggests that NNIP can accurately describe the bond-breaking and bond-forming processes.

\section{E. Hydrogen-surface reaction}

The interactions of hydrogen with surfaces are always engaged in the topic of $\mathrm{HE}$, such as the hydrogen charge and uncharge process, nanoscale cavity migration in a $\mathrm{H}_{2}$-gas environment [82]. The adsorption of $\mathrm{H}$ atom or $\mathrm{H}_{2}$ molecule, and $\mathrm{H}_{2}$ molecule formation and dissociation processes in iron surfaces has been extensively studied using DFT [83-86]. However, the description of these process remains difficult for the empirical potentials. The adsorption energies of a $\mathrm{H}$ atom on several low-index surfaces were reproduced with NNIP and show good agreement with the DFT results; these results are tabulated in Table S6 in the Supplemental Material [27]. Subsequently, the $\mathrm{H}_{2}$ molecule dissociation process on the (001) surface is further demonstrated via CI-NEB calculations. The potential energy profile along the formationdissociation path and several typical atomic configurations are shown in Fig. 3(b).

To compute the $\mathrm{H}_{2}$ formation-dissociation energetics and pathway, we used a supercell with a size of $3[100] \times 3[010] \times$ 6[001] and comprising $108 \mathrm{Fe}$ atoms. In the case of two $\mathrm{H}$ atoms on the (001) surface [see Fig. 3(b), A], both DFT and NNIP predicted that the configuration of those $\mathrm{H}$ atoms at hollow sites with a separation of $\mathrm{d}_{\mathrm{H}-\mathrm{H}}=\sqrt{2} a_{0}$ ( $a_{0}$ is the lattice constant) is energetically preferred. Thus, this configuration is set as the initial state. The final configuration is obtained by relaxing the configuration with the two $\mathrm{H}$ atoms at $6.0 \AA$ above the surface [see Fig. 3(b), E]. H atoms present two state transitions in the formation-dissociation process: the transition state from state A to state $\mathrm{C}$ and the transition state from state $\mathrm{C}$ to state E. Notably, the NNIP predicts the distances of $\mathrm{H}$ atoms at state B and state D as 1.33 and $2.36 \AA$, respectively; this closely matches the DFT results of 1.4 and $2.2 \AA$ [86], respectively. The energy barrier of the transition from state A to state $\mathrm{C}$ is $1.090 \mathrm{eV}$, which is introduced by the breaking of two $\mathrm{Fe}-\mathrm{H}$ bonds, for which the bond energy obtained with DFT is $0.648 \mathrm{eV}$ [87], and forming the $\mathrm{H}-\mathrm{H}$ bond. The $\mathrm{H}_{2}$ molecule at state $\mathrm{C}$ is $0.175 \mathrm{eV}$ stabilized with respect to the free $\mathrm{H}_{2}$ molecule at state $\mathrm{E}$; this energy is termed molecular adsorption energy $\left(E_{\text {ads }}^{\mathrm{H}_{2}}\right)$ and matches both the DFT result of $0.186 \mathrm{eV}$ [87] and the experimental result of $0.187 \mathrm{eV}$ [88]. The transition from state $\mathrm{C}$ to state $\mathrm{E}$ has the energy barrier of $0.268 \mathrm{eV}$. The energy barrier for $\mathrm{H}_{2}$ molecule transition from state $\mathrm{E}$ to state $\mathrm{C}(\mathrm{D})$ is about $0.1 \mathrm{eV}$, indicating the repulsive interaction between the molecule and substrate. A movie for the $\mathrm{H}_{2}$ molecule formation process on the (001) surface at $800 \mathrm{~K}$ can be found in the Supplemental Material and is named Movie S2 [27].

Note that most of the above hydrogen-surface reaction data are not included in the reference database, while structures from AIMD for low-index surfaces (see Table S2 in the Supplemental Material [27]) with various number of $\mathrm{H}$ atoms are included in the reference database.

\section{F. Hydrogen-grain boundaries interaction}

Hydrogen segregation and diffusion along GBs are crucial for H-induced intergranular cracking [89-95]. The $\Sigma 5(310)[001]$ symmetric tilt GB (hereafter referred to as $\Sigma 5$ GB) is adopted to demonstrate the quality of NNIP for studying the $\mathrm{H}$ segregation and diffusion along GBs. The employed $\Sigma 5$ GB supercell has a size of $2[001] \times 1[1 \overline{3} 0] \times 3[310]$, and thus contains a total of $80 \mathrm{Fe}$ atoms, as shown in Fig. 4(a).

First, the solution energy of hydrogen to interstitial sites [see Fig. 4(a)] in $\Sigma 5 \mathrm{~GB}\left(E_{\mathrm{s}}^{\mathrm{GB}}\right)$ was calculated by Eq. (6). NNIP predicted that site 1 (short for $s 1$, the same below), which is the equivalent of $s 4$, is the most stable solution site having an $E_{\mathrm{s}}^{\mathrm{GB}}$ of $-0.180 \mathrm{eV}$; this well agrees with the DFT result of $-0.187 \mathrm{eV}[96,97]$. The solution energies for other sites are presented in Fig. 4(b) and are found to align well with the results of our DFT calculations, although the solution energies were not fully included in the reference database.

The energy profile of $\mathrm{H}$-atom diffusion toward the GB and in the GB was studied via CI-NEB calculations, which was not included in the reference database. The following three diffusion paths were considered: (a) between two adjacent most stable solution sites in the [001] direction, i.e., $s 1 \leftrightarrow s 1^{[001]}$; (b) from the most stable solution site to a $\mathrm{T}$ site in bulk, i.e., $s 4 \leftrightarrow s 8$; and (c) along the entire GB, i.e., $s 1 \leftrightarrow s 2 \leftrightarrow s 3 \leftrightarrow s 4$.

Each of the above-mentioned three paths has been reported in previous studies [91,96], with the exception of the part of $s 2 \leftrightarrow s 3 \leftrightarrow s 4$ in path (c). The unavailable part in path (c) was newly calculated in this study. The diffusion energy barrier for path (a) with NNIP is $0.237 \mathrm{eV}$, which well agrees with the reported DFT calculations of $0.250 \mathrm{eV}[91,96]$. For the second path, two humps in the diffusion path yield energy barriers of 0.451 and $0.568 \mathrm{eV}$, respectively, which also agree with the previously reported DFT results of 0.40 and $0.57 \mathrm{eV}$ [96]. The diffusion pathway of path (c) is shown in Fig. 4(c). NNIP can properly describe the entirety of the migration process. The large energy barrier between $s 2$ and $s 3$ of $0.674 \mathrm{eV}$ denotes that it is extremely difficult for the $\mathrm{H}$ atom to migrate across the entire GB. The energy barrier from $s 4$ to $s 6$ with NNIP is $0.165 \mathrm{eV}$, i.e., significantly lower than that of $s 2$ to $s 3$. The trapped hydrogen atom prefers to stay in the region enclosed by the sites of $s 4$ and $s 6$ and their equivalent sites in the [001] and [310] directions. This qualitatively meets the conclusion in the previous study [91].

The effects of $\mathrm{H}$ atoms on $\mathrm{GB}$ decohesion were further tested and compared with DFT results and shown in the Supplemental Material (Sec. SIX) [27]. 

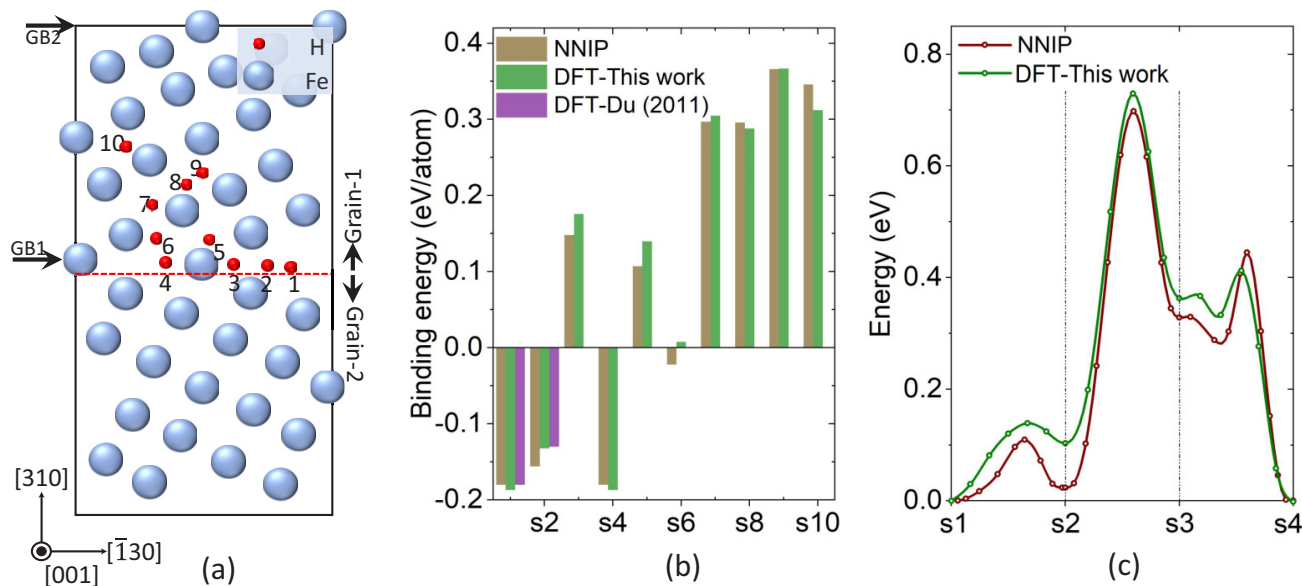

FIG. 4. (a) Atomic structure of $\Sigma 5$ GB showing several sites of interest at and around the GB plane. Red dotted line indicates the predesigned fracture path, and grain-1 and grain-2 represent two separated grains after fracturing. (b) Solution energies of $\mathrm{H}$ atom at various sites $\left(E_{\mathrm{s}}^{\mathrm{GB}}\right)$ presented in (a). (c) Diffusion barriers of the $\mathrm{H}$ atom along the [130] direction in the GB plane. DFT results extracted from Ref. [96] are plotted in (b).

\section{G. Hydrogen-screw dislocation interaction}

The presence of hydrogen around screw dislocation is believed to change its motion because the trapping, such as a segregation isotherm, stabilizes the screw dislocation by reducing total free energy. Therefore, the solution energies of the $\mathrm{H}$ atom at various sites around the screw dislocation (using the large PAD model described in Sec. III B and presented in Fig. S5(a) in the Supplemental Material [27]) should be reproduced with NNIP. Using NNIP we calculated them and compared with the available DFT results, as shown in Figs. 5(a)-5(c). Note that DFT data of the hydrogen-screw dislocation interaction using the small 208-Fe-atom model with an easy core configuration at the center (see Fig. S2(d) in the Supplemental Material [27]) are included in the reference database. The positions of Fe atoms in the outer layers are fixed to sustain the core structure of dislocation. The $\mathrm{H}$ atom is placed at various sites around the dislocation to represent the different binding sites in Fig. 5(a). The H-atom solution energy in the screw dislocation calculated with NNIP is shown in Fig. 5(c). The energetically favorable sites can be found as $E_{1}$ and $E_{2}$. The solution energy for the $\mathrm{H}$ atom at which the site of $\mathrm{C}_{m}(m=1-3)$, is $0.13 \mathrm{eV}$; nonetheless, it is still energetically preferred over bulk sites. All solution energies are within small error ranges compared with the DFT calculations in Ref. [98] [see Fig. 5(c)].

The diffusion of interstitial solute along the screw dislocation is also of interest [99] because the trapped $\mathrm{H}$ atoms may mainly diffuse along the screw dislocation (this diffusion mechanism is called pipe diffusion). Thus, the diffusion barriers around the screw dislocation determine the diffusion pathway and diffusivity along the screw dislocation. As expected in Ref. [98], the region enclosed by $E_{1}$ and $E_{2}$ is an energy basin that the $\mathrm{H}$ atom can move with a tiny barrier of $0.048 \mathrm{eV}$. The barrier for an $\mathrm{H}$ atom to enter the core region of dislocation from the energy basin is $0.15 \mathrm{eV}$, whereas that to diffuse along the dislocation inside the core is only $0.057 \mathrm{eV}$, i.e., much lower than the path from the $E_{1}$ site to the neighboring $E_{1}$ site along the Burgers vector, which is
$0.638 \mathrm{eV}$. The actual diffusion path at finite temperature can be observed in MD simulation for the random walking $\mathrm{H}$ atoms. This is shown in the following application section, whereas other considered diffusion barriers are shown in Fig. 5(d). Data on $\mathrm{H}$-atom diffusion in a model with screw dislocation are not included in the reference database.

The effect of $\mathrm{H}$ on the core shape and mobility of screw dislocation can be indirectly described using the $\gamma$ surface. In the presence of an $\mathrm{H}$ atom at the $\mathrm{T}$ site in the sliding interlayer, the maximum of the $\gamma$ surface along the $\langle 111\rangle$ direction is $5 \mathrm{meV} / \AA^{2}$ lower than that of pure iron, as shown in Fig. 5(e), which is consistent with the DFT results [100]. The calculated kink pair nucleation energy at zero external stress for dislocation with one $\mathrm{H}$ atom at the site of $E_{\mathrm{k}}$ [see Fig. 5(a)] located in dislocation gliding direction [112] is displayed in Fig. 5(f). We find that the nucleation energy is lowered because of the attractive interaction between screw dislocation core and solute $\mathrm{H}$ atom located in the gliding direction [inset of Fig. 5(f)], which is qualitatively consistent with the prediction using the LT+DFT model [98]. No data regarding the kink pair nucleation energy are included in the reference database. The reduction in the maximum of the $\gamma$ surface along the $\langle 111\rangle$ direction suggests that the presence of an $\mathrm{H}$ atom enhances the intrinsic mobility of dislocation; however, simultaneously the dragged effect by the presence of $\mathrm{H}$ atoms at the dislocation core should be considered, which may depress the mobility of dislocation and depends on the $\mathrm{H}$ concentration and temperature [101]. This requires further study.

\section{APPLICATIONS}

Hydrogen-enhanced decohesion (HEDE) [102], hydrogenenhanced localized plasticity (HELP) [103], hydrogenenhanced strain-induced vacancies (HESIVs) [104], and other HE mechanisms have been proposed in the literature [105], and posterior interpretations of experimental observations have been made. Nevertheless, the physics of HE remains poorly understood. It has been suggested that all hydrogen- 


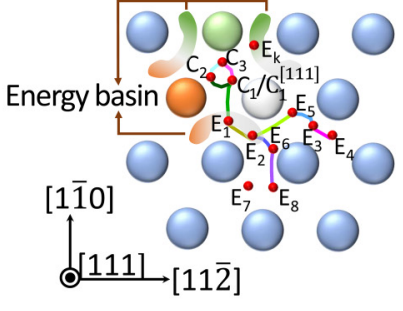

(a)

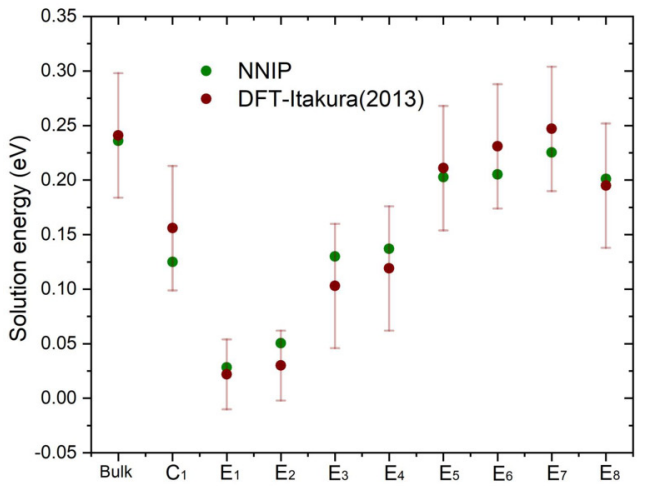

(c)

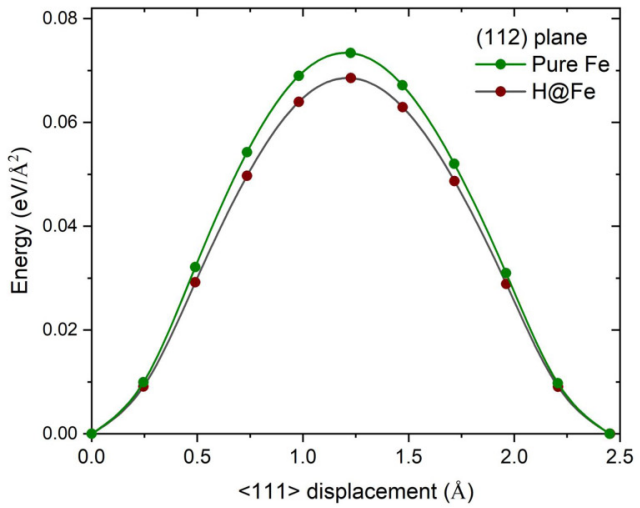

(e)

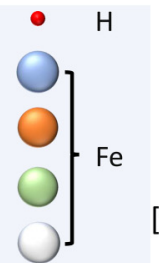

[111]

[112]

(b)

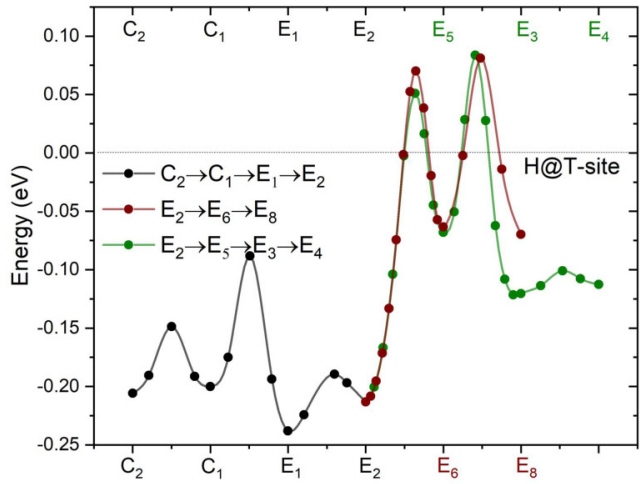

(d)

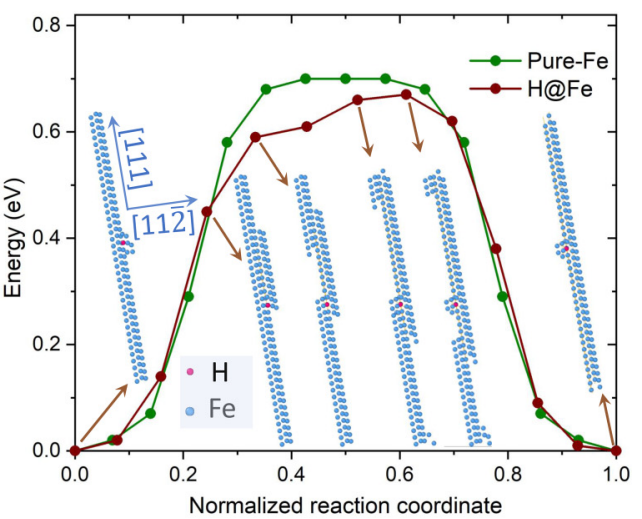

(f)

FIG. 5. (a) Positions of $\mathrm{H}$ solution sites around the easy core configuration of screw dislocation. The $E_{\mathrm{k}}$ site is equivalent to $E_{1}$ and is used to calculate the influence of the $\mathrm{H}$ atom on kink pair nucleation energy (see text for details). The energy basins are shaped like curvy caterpillars. The side view of (a) is displayed in (b). (c) Solution energies of the H atom at various sites marked in (a) and T site in bulk, presented with DFT results from Ref. [98]. (d) Diffusion barriers of $\mathrm{H}$ atom in the paths marked in (a); the solution energy of the $\mathrm{H}$ atom at the $\mathrm{T}$ site is set as the benchmark, which is shown by the dotted line. (e) $\gamma$ surface of pure iron with and without $\mathrm{H}$ atom. (f) Kink nucleation energy of dislocation with and without $\mathrm{H}$ atom (at $E_{\mathrm{k}}$ ).

induced embrittlement processes cannot be driven by any single mechanism [106]. Therefore, this section demonstrates several key phenomena concerning the HE, such as hydrogen charging and diffusion to defective $\alpha$-iron, temperaturedependent hydrogen trapping and desorption to different defects in $\alpha$-iron, and plasticity and hydrogen-enhanced GB fracture by MD simulations with the constructed NNIP.

\section{A. Hydrogen charging and diffusion processes in GB and screw dislocation}

The first step of $\mathrm{H}_{2}$ gas permeation into metals is dissociation of $\mathrm{H}_{2}$ molecules on the metal surface. $\mathrm{H}_{2}$ molecules also dissociate and form on the surfaces of crack tips and nanocavities, which are all critical processes for HE. In this section, the charging process and diffusion behaviors of $\mathrm{H}$ atoms in tilt GB and screw dislocation of $\alpha$-iron are simulated.

The hydrogen charging and diffusion behavior in a tilt GB were simulated using a bicrystal model with a $\Sigma 5$ symmetric tilt GB [see Fig. 6(a)]. The supercell used in this simulation contains $4805 \mathrm{Fe}$ atoms and has the size of $5 a_{T}, 4 \sqrt{10} a_{T}$, and $6 \sqrt{10} a_{T}$ in the [001], [13̄0], and [310] directions, where $a_{\mathrm{T}}$ is the lattice constant at temperature $T$. An additional vacuum region with thickness of $30 \AA$ was set in the [1 $\overline{3} 0]$ direction to enable the injection of $\mathrm{H}_{2}$ gas. Periodic boundary conditions 


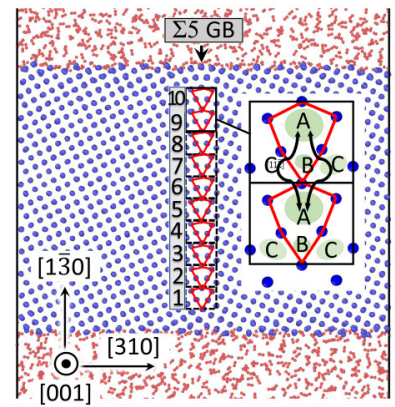

(a)

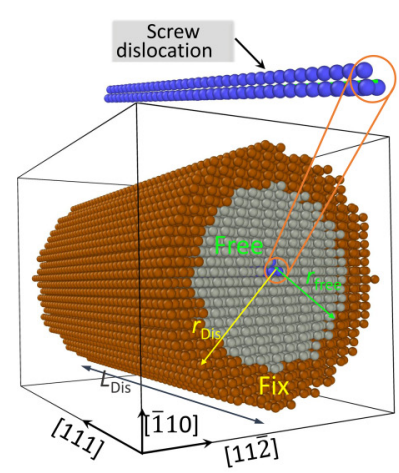

(d)

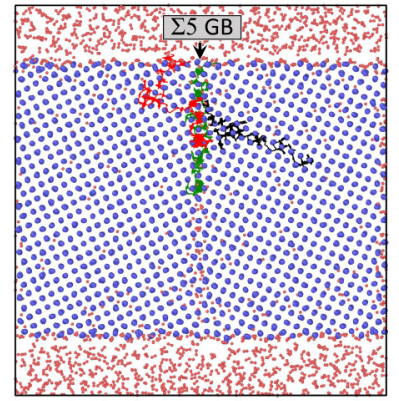

(b)

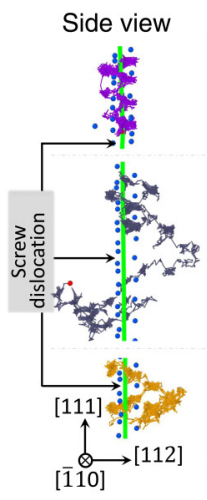

(e)

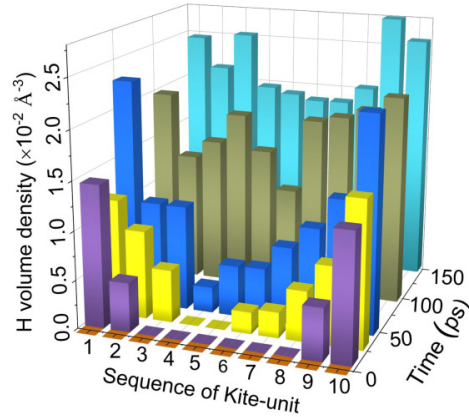

(c)

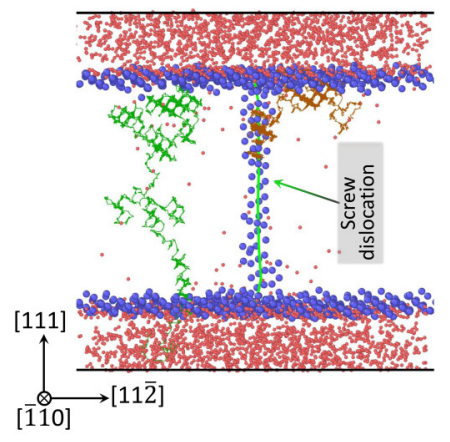

(f)

FIG. 6. (a) Snapshots of the system at the simulation time of 0 ps. Several kite units are designed in the [1 $\overline{3} 0]$ direction and their sequence number is marked next to it individually. The large view of kite unit and H-atom diffusion path is also presented. (b) Snapshot at 150 ps. Trajectories of $\mathrm{H}$ atoms of interest, i.e., atoms $\mathrm{H}_{\mathrm{O}}$ (olive line) and $\mathrm{H}_{\mathrm{R}}$ (red line), at the simulation time interval of $0-40$ ps, $\mathrm{H}_{\mathrm{B}}$ (black line) at 100-130 ps are presented. (c) $\mathrm{H}$ volume density $\left(\rho_{\mathrm{H}}\right)$ evolution with time in each kite unit marked in (a). (d) The screw dislocation model used in the simulation. The fixed and free Fe atoms are indicated by dark red and dark gray balls, respectively, and the three columns of blue balls denote the dislocation core. (e) Three typical trajectories of $\mathrm{H}$ atoms, presented in a MD simulation for 130 ps and viewed from two directions. (f) Interior trajectories of $\mathrm{H}$ atoms in the $\mathrm{H}$-charging simulation; all iron atoms are set to be invisible. The thick green line in (d)-(f) indicates the dislocation line obtained by the DXA tool implemented in the OVITO code [107].

were employed in all directions. To quantitatively describe the penetration depth of the $\mathrm{H}$ atom, several kite units along the $[1 \overline{3} 0]$ direction were designed [see Fig. $6(\mathrm{a})]$ and the $\mathrm{H}$ volume density $\left(\rho_{\mathrm{H}}\right)$ in individual kite units was calculated at different MD stages. Each kite unit can be divided into four regions: $\mathrm{A}, \mathrm{B}$, and $2 \times \mathrm{C}$, as marked in the inner panel of Fig. 6(a). The simulation was conducted as follows:

(a) Relax the clean GB supercell at $600 \mathrm{~K}$ for 2 ps with a time step of $1 \mathrm{fs}$.

(b) Inject $\mathrm{H}_{2}$ molecules into the vacuum with a time step of $0.2 \mathrm{fs}$.

(c) Equilibrate the system for 0.2 ps on NPT ensemble with a pressure of $2 \mathrm{GPa}$ in the [130] direction.

(d) Perform MD runs and calculate the average for $\rho_{\mathrm{H}}$ estimation.

Figure 6(b) shows the snapshot of the bicrystal model simulated for $150 \mathrm{ps}$. The figure reveals that the $\mathrm{H}$ atoms prefer diffusing along the GB and the $\mathrm{H}$ concentration in the GB is, as expected, significantly higher than that in the bulk region. The time evolution of $\rho_{\mathrm{H}}$ is shown in Fig. 6(c) and suggests that $\mathrm{H}$ atoms gradually diffuse from the two surfaces to the interior part; i.e., $\mathrm{GB}$ is a ditch for $\mathrm{H}$ transportation. The $\mathrm{H}$ diffusion path can be directly depicted using the $\mathrm{H}$ traveling trajectories, and three typical diffusion manners are shown in Fig. 6(b). Olive, red, and black lines show the trajectories of atoms $\mathrm{H}_{\mathrm{O}}, \mathrm{H}_{\mathrm{R}}$, and $\mathrm{H}_{\mathrm{B}}$, respectively. $\mathrm{H}$ atoms can diffuse from the surface to the interior part through the GB, as observed for $\mathrm{H}_{\mathrm{O}}$. $\mathrm{H}$ atoms can also first enter the bulk region and diffuse in the way of a random walk, as observed for $\mathrm{H}_{R}$. Subsequently, they are trapped by the GB and diffuse similar to $\mathrm{H}_{\mathrm{O}}$. This fact shows that $\mathrm{H}$ can diffuse faster in the bulk than along the GB. The diffusion of $\mathrm{H}$ atoms along the GB occurs throughout the entirety of the (310) plane, including both [13̄0] and [001] directions. The diffusion path in the [001] direction is the path I mentioned in Sec. III F. The trajectory in the [1 $1 \overline{3} 0]$ direction is given as $\mathrm{H}_{\mathrm{O}}$ in Fig. 6(b). This trajectory indicates that $\mathrm{H}$ atoms tend not to diffuse directly through the GB unit but instead deviate to region $\mathrm{C}$ before jumping to region $\mathrm{A}$ in the next kite unit, as schematically illustrated in the subpanel of Fig. 6(a). With an increase in hydrogen concentration in the GB region, the GB serves as a source of hydrogen migration into the bulk, such as the migration path of $\mathrm{H}_{\mathrm{B}}$ presented in Fig. 6(b). Additionally, hydrogen segregation at and diffusion along the $\mathrm{GB}$ is a process strongly relying on the angle between grains [89]; one can directly observe the hydrogen diffusion path in any other GBs via this charging model with the NNIP.

The diffusion of $\mathrm{H}$ atoms in screw dislocation was further studied. The atomic model used in simulation is shown in Fig. 6(d). The radius of the atomic model in the (111) plane $\left(r_{\text {Dis }}\right)$ and the length of the atomic model in the [111] direction 
$\left(L_{\text {Dis }}\right)$ are $31.2 \AA$ and $40.1 \AA$ respectively, with a total of $13920 \mathrm{Fe}$ atoms. The radius of the free region $\left(r_{\text {free }}\right)$ is $26.2 \AA$. First, to see individual $\mathrm{H}$-atom diffusion around the screw dislocation core, a single $\mathrm{H}$ atom was placed at different positions in the screw dislocation core. Thereafter, MD simulations were performed at $600 \mathrm{~K}$ on an NVT ensemble for 130 ps. As the results, the following three typical diffusion motions of $\mathrm{H}$ atoms were observed [Fig. 6(e)]:

(1) The first type of H-atom motion, indicated with purple trajectory, exhibited a spiral motion along the Burgers vector of dislocation and was frequently pulled out from the core region to the energetically preferred sites (energy basin).

(2) The second type of $\mathrm{H}$-atom motion, indicated with the dark gray trajectory, exhibited the same diffusion path as the purple one, but it eventually escaped the constraints of the energy basin and performed a random walk in the bulk region.

(3) The third type of H-atom motion, indicated with the yellow trajectory, exhibited that such random walking atoms in the bulk region are susceptible to being recaptured by the dislocation.

To obtain a more general diffusion path of an $\mathrm{H}$ atom in a system featuring dislocation, a simulation similar to the above-mentioned $\mathrm{H}$ charging of the $\mathrm{GB}$ was conducted. In this simulation, the atomic model sizes of $r_{\text {Dis }}, L_{\text {Dis }}$, and $r_{\text {free }}$ are $42.5,40.1$, and $32.5 \AA$, respectively, with a total of 20301 $\mathrm{Fe}$ atoms. A vacuum region with a thickness of $10 \AA$ was set in the [111] direction to enable the injection of $\mathrm{H}_{2}$ gas. The injected $\mathrm{H}_{2}$ molecules were constrained within a cylindrical region in the [111] direction centered at the core location of the dislocation, taking the radius as the free iron region. $\mathrm{H}$-atom diffusion was enhanced by gradually increasing the quantity of the $\mathrm{H}_{2}$ molecules in that vacuum region. The trajectories of the diffused atoms are displayed in Fig. 6(f) and Fig. S11 in the Supplemental Material [27]. Although a diffusion path inside the dislocation core along the Burgers vector is formed [dark red trajectory in Fig. 6(f) and in Figs. S11(a)-S11(c)], more atoms prefer diffusing through the bulk region via random walking ([green trajectory in Fig. 6(f) and Figs. S11(d)-S11(p)], and H-atom diffusion is faster in bulk than that in dislocation [Fig. 6(f) and Fig. S11]. After carefully checking the snapshots in the charging process, we further uncovered that the screw dislocation traps random-walking $\mathrm{H}$ atoms and those $\mathrm{H}$ atoms can be transported along the screw dislocation line through repeated trapping and detrapping from the screw dislocation core. However, hydrogen transportation efficiency along a screw dislocation was really poor because of the higher solution energy in the dislocation core relative to the basin region, frequently causing trapped $\mathrm{H}$ atoms to be pulled out from the core region and kept in the energy basin.

\section{B. Hydrogen trapping and desorption at defects}

Understanding the hydrogen distribution in defects not only helps to characterize defects in metals [108], but can also guide the artificial creation of defects designed to trap and reduce mobile $\mathrm{H}$ atoms in metals. However, the hydrogen concentration in and around defects (e.g., vacancies, GBs, and screw dislocation) in $\alpha$-iron at various temperatures remains an open question.
Combining the grand canonical Monte Carlo (GCMC) method [109] with MD simulations (the MD/GCMC hybrid method), we studied the radial distribution function of hydrogen volume density $\left(\rho_{H}^{V}\right)$ around various defects. Herein, to adjust the hydrogen bulk concentration in $\alpha$-iron to approximately 40 atomic ppm (appm), the $\mathrm{H}$-atom chemical potential was set to $-2.16,-2.25,-2.37,-2.48$, and $-2.59 \mathrm{eV}$ at 200 , $300,400,500$, and $600 \mathrm{~K}$, respectively. A $12 \times 12 \times 12$ supercell with one vacancy at the center, 3455 iron atoms in total, was employed as the vacancy model, and the models used in Sec. IV A were adopted for the GB and screw dislocation (the one with $13920 \mathrm{Fe}$ atoms) cases without inserting the vacuum region. $100 \mathrm{GCMC}$ trials were conducted in each $10 \mathrm{MD}$ steps with a time step of $0.5 \mathrm{fs}$.

To analyze the radial distribution function (RDF) of the volume density of the number of $\mathrm{H}$ atoms, spherical shell regions co-centered with the vacancy, slab regions with equivalent location above and below the GB interface, and cylindrical shell regions coaxed with the screw dislocation are set respectively for the models of vacancy, GB, and screw dislocation. The thickness of each region (i.e., the mesh size of the RDF analysis) was $2 \AA$. The $\rho_{H}^{V}$ in an individual region was determined as follows:

$$
\rho_{H}^{V}=\frac{1}{N} \sum_{i=1}^{N} \frac{n_{\mathrm{H}}}{V_{\text {shell } / \text { slab }}},
$$

where the $n_{\mathrm{H}}$ and $V_{\text {shell/slab }}$ denote the number of $\mathrm{H}$ atoms and the volume of an individual shell or slab region, respectively. These values were averaged over $5 \times 10^{6}$ GCMC trials in 50000 MD steps.

As shown in Fig. 7(a), the trapped $\mathrm{H}$ atoms were distributed within a very small region (within $2 \AA$ ) around the defects at all considered temperatures. An exception appeared in the case of screw dislocation [inner panel of Fig. 7(a)]; after carefully checking snapshots, this wider distribution was contributed by the $\mathrm{H}$ atoms located at $E_{3}$ and $E_{4}$ [Fig. 5(a)]. Naturally the number of trapped $\mathrm{H}$ atoms decreased with increasing temperature in all defects. Snapshots of typical hydrogen distributions at various temperatures can be found in the Supplemental Material (Fig. S12) [27]. The temperaturedependent desorption rate, such as the quantity reduction of the trapped $\mathrm{H}$ atoms with respect to temperature, $-\partial \rho_{\mathrm{H}}^{\mathrm{V}} / \partial T$, is plotted in Fig. 7(b).

For the vacancy case, the $\rho_{\mathrm{H}}^{\mathrm{V}}$ was $0.15 \mathrm{H} / \AA^{3}$ at $200 \mathrm{~K}$, equivalent to $5.01 \mathrm{H}$ per vacancy. The $\rho_{\mathrm{H}}^{\mathrm{V}}$ gradually decreased from $0.14 \mathrm{H} / \AA^{3}$ to $0.06 \mathrm{H} / \AA^{3}$ as the temperature increased from 300 to $500 \mathrm{~K}$ (equivalent to a desorption of three $\mathrm{H}$ atoms per vacancy). The desorption peak presented around $400 \mathrm{~K}$ in Fig. 7(b) matches the peak obtained at $400-440 \mathrm{~K}$ in experiment [110].

For the GB case, at $200 \mathrm{~K}, \mathrm{H}$ atoms were trapped in regions A and C [Fig. 6(a)]. As shown in Sec. III F, the solution energy of an $\mathrm{H}$ atom at $s 5$ (corresponding to region $\mathrm{C}$ ) is positive at $0 \mathrm{~K}$, but is still $0.2 \mathrm{eV}$ lower than at the $\mathrm{T}$ site in bulk. The desorption of these $\mathrm{H}$ atoms gives rise to the peak at $300-400 \mathrm{~K}$ in Fig. 7(b), which aligns with the experimental desorption peak at $400 \mathrm{~K}$ ascribed to the desorption of $\mathrm{H}$ atoms released from GBs [111]. 


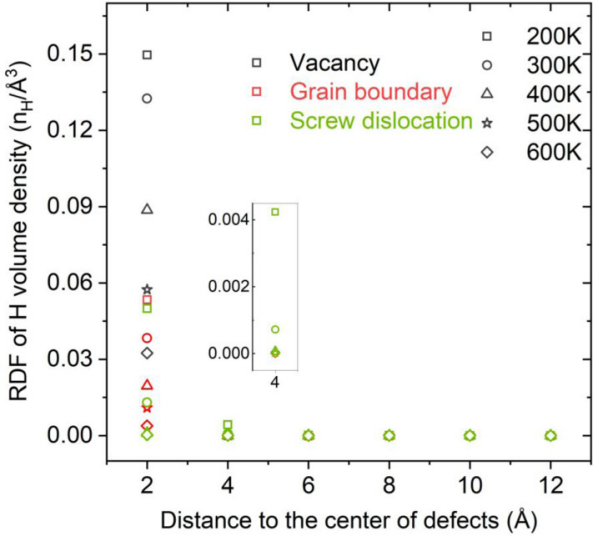

(a)

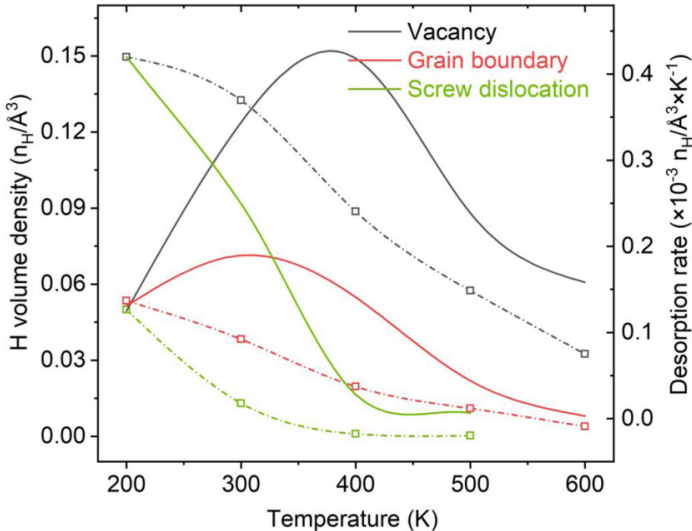

(b)

FIG. 7. (a) Radial distribution function (RDF) of volume densities of the number of $\mathrm{H}$ atoms around vacancy, GB, and screw dislocation at various temperatures. (b) Volume density of the number of $\mathrm{H}$ atoms around defects within $2 \AA$ [dotted lines, replotting of the first column data of (a)] and the hydrogen desorption rates at various temperatures (solid lines).

For a screw dislocation model with a length of $1 b$, there are three energy basins, each lying between two neighboring core atoms [Fig. 5(a)]. At $200 \mathrm{~K}$, each energy basin can accommodate at most $1 \mathrm{H}$ atom. Importantly, the desorption peak should exist below $300 \mathrm{~K}$ at least [see Fig. 7(b)]. This argument might explain the experimental desorption peak observed at $220 \mathrm{~K}$ [111].

Before closing this section, we would like to emphasize that any trapped $\mathrm{H}$ atoms can convert to mobile atoms at higher temperatures. This hydrogen state transition is critical for the understanding and prevention of HE.

\section{MD-GCMC tensile test for $\mathrm{H}$-charging bicrystal model with twist GB}

Applying MD with EIP, Wan et al. recently proposed a fresh model for HE. They suggested that GB activation by the GB-dislocation reaction is a key component in the HE process of metals [106]. Nevertheless, the quantitative behavior must be validated by DFT calculations to ensure the accuracy of the result [112]. Here, MD-GCMC hybrid tensile tests for a twist grain boundary with and without hydrogen charging were performed to create the connects among hydrogen charging, GB activation, GB-dislocation reaction, and crack nucleation and propagation at DFT accuracy.

Before performing tensile tests, the twist GB formation energies $\left(\gamma_{\text {twist }}\right)$ of $\Sigma 9\{1 \overline{1} 0\}$ and $\Sigma 5\{100\}$, with 288 and $60 \mathrm{Fe}$ atoms, respectively, were calculated. The $\gamma_{\text {twist }}$ of the $\Sigma 9\{1 \overline{1} 0\}$ twist GB obtained with NNIP is $0.95 \mathrm{~J} / \mathrm{m}^{2}$ and shows perfect agreement with the DFT result of $0.98 \mathrm{~J} / \mathrm{m}^{2}$. The $\gamma_{\text {twist }}$ of the $\Sigma 5\{100\}$ twist GB with NNIP is $2.21 \mathrm{~J} / \mathrm{m}^{2}$, which well agrees with the reported DFT result of $2.12 \mathrm{~J} / \mathrm{m}^{2}$ [52] and our DFT result of $2.29 \mathrm{~J} / \mathrm{m}^{2}$. The performance of NNIP for twist GB shows its good transferability because no twist data were included in the reference database.

The $\Sigma 9\{1 \overline{1} 0\}$ twist GB (hereafter referred to as $\Sigma 9$ twist or twist) was adopted for the tensile test. The dimension of the H-free clean $\Sigma 9$ twist is $51.2,48.2$, and $96.9 \AA$ in [114], [221] , and [110] directions, respectively; periodic boundary conditions were employed in all directions, and the model contained a total of $20736 \mathrm{Fe}$ atoms. The twist model is illustrated in Fig. 8(a).

Two comparison tensile tests were performed for the clean and $\mathrm{H}$-charging twists. A constant MD tensile strain rate of $1 \times 10^{9} \mathrm{~s}^{-1}$ in the [110] direction and the temperature of $300 \mathrm{~K}$ were adopted for both tensile tests. The time steps of 1.0 and $0.5 \mathrm{fs}$ were used in the tensile tests for the clean and $\mathrm{H}-$ charging twists, respectively. For the clean twist, normal MD simulation on an NPT ensemble with constant tensile engineering strain rate was employed. For the $\mathrm{H}$-charging twist, $\mathrm{H}$ atoms were introduced via the GCMC method with a constant $\mathrm{H}$ chemical potential of $-2.25 \mathrm{eV}$ (bulk $\mathrm{H}$ concentration is 40 appm at $300 \mathrm{~K}$ ) during MD simulation on NVT(MD) and $\mu \mathrm{VT}(\mathrm{GCMC})$ ensembles. Therefore, $\mathrm{H}$ atoms can be inserted in and extracted from the supercell, and then the Fe-H system may approach an equilibrium state. 500 GCMC trials were conducted every 0.05 ps of MD simulation; finally, approximately $340 \mathrm{H}$ atoms were introduced into the twist model within 100 ps of MD simulation. Then, the MD-GCMC hybrid tensile test on NPT and $\mu \mathrm{VT}$ ensembles was conducted, while the lateral dimensions of the model were adjusted according to the zero-pressure conditions. Note that time cannot be real in this simulation because GCMC trial steps were inserted during the MD simulation. However, for simplicity, here we measured time according to the number of MD steps, thus assuming that $\mathrm{H}$ concentration is always nearly equilibrium.

The clean twist model exhibits elastic deformation when the tensile engineering strain is lower than $14.0 \%$, as shown in the left panel of Fig. 8(b). Its stress starts to decrease after reaching the maximum of $21 \mathrm{GPa}$ at a strain of $14.5 \%$. The release of the stress was introduced by the emission of dislocation, as shown in the middle panel of Fig. 8(b); the dislocation volume density ( $\rho_{\text {Dis }}$, dislocation length divided by the volume of the model) sharply increased from 0 to $2.8 \times 10^{-3} \AA^{-2}$ at that strain. Several snapshots in the tensile test are shown in Fig. 8(d).

The H-charging twist model was also subject to elastic deformation within the tensile stain of $11 \%$. The initiation of plastic deformation of the $\mathrm{H}$-charging twist was presented around the strain of $12.0 \%$ with a stress of $17 \mathrm{GPa}$, which is 

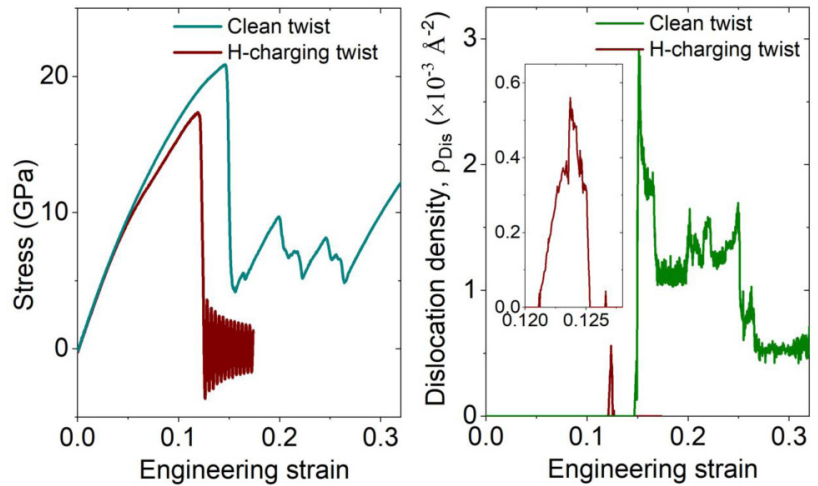

(b)

Clean twist (MD)

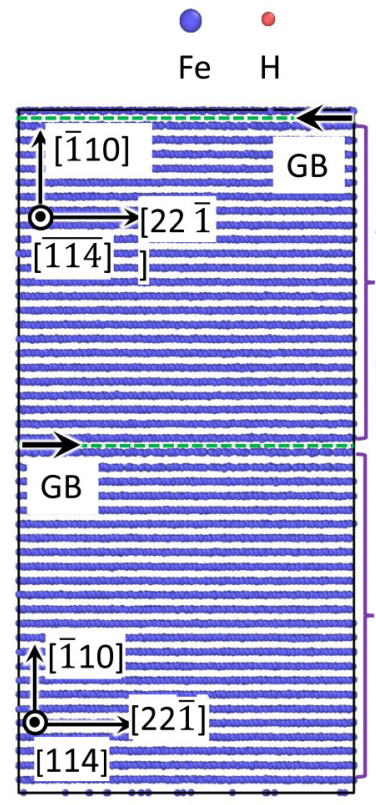

(a)
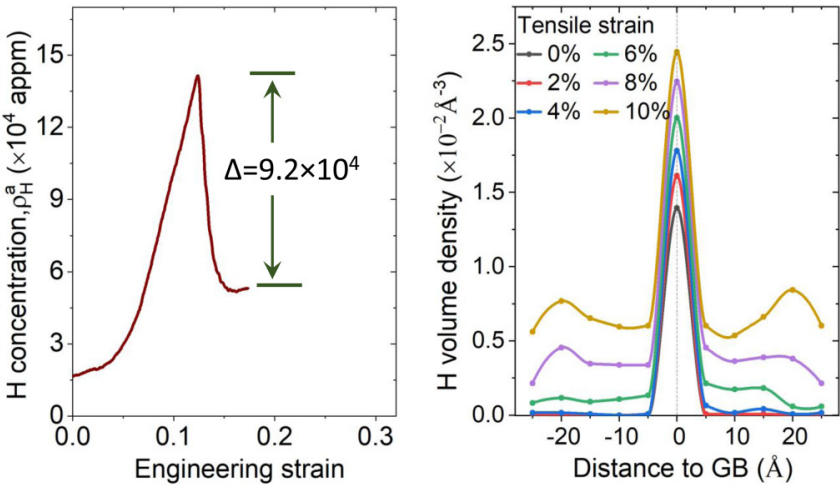

(c)
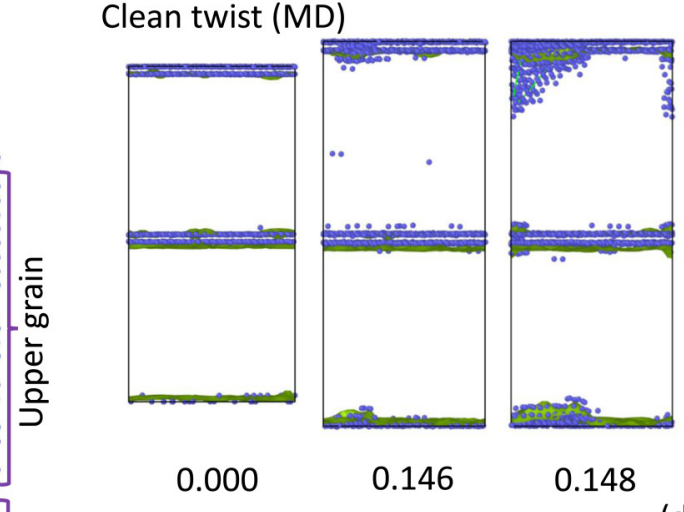

0.148

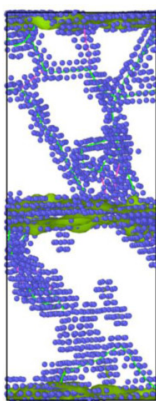

0.156

(d)

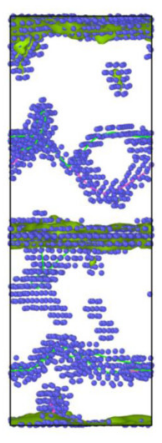

0.220

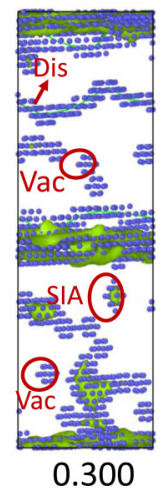

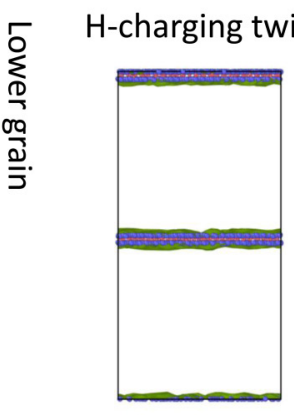

0.000

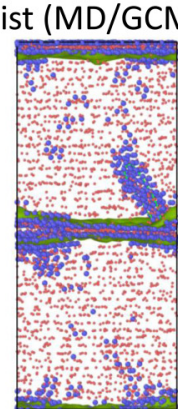

0.122

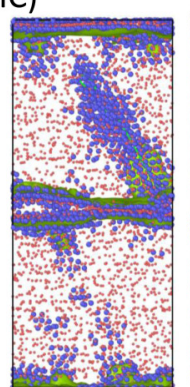

0.123

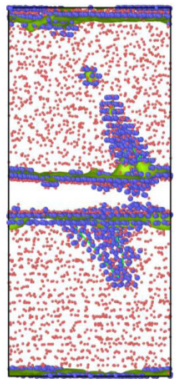

0.125

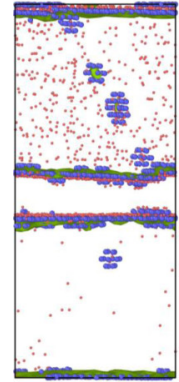

0.140

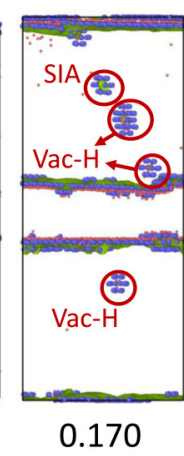

(e)

FIG. 8. Tensile tests for clean and H-charging $\Sigma 9$ twists. (a) Clean $\Sigma 9$ twist model. (b) Left, middle, and right panels present the tensile stress, dislocation volume density $\left(\rho_{\text {Dis }}\right)$, and $\mathrm{H}$ atomic concentration $\left(\rho_{\mathrm{H}}^{a}\right)$ in the models versus the tensile strain. (c) Volume density of $\mathrm{H}$ $\left(\rho_{\mathrm{H}}^{V}\right)$ in the H-charging twist at various tensile strains. (d), (e) Snapshots of clean and H-charging twists at various tensile strains (Fe atoms with bcc lattice are set as invisible), and the corresponding strains are printed under each panel. Blue and red dots denote Fe and $\mathrm{H}$ atoms, respectively. The green surface and thick lines in (d) and (e) are the regions that differ from bcc lattice [113] and dislocations, respectively, which are analyzed by the DXA tool implemented in the OVITO code [107].

lower than that of the clean twist. As presented in the snapshot $(0.0 \%)$ in Fig. 8(e), even before applying tensile strain, the $\mathrm{H}$ concentration in the GB region was much higher than that in the bulk, i.e., $\mathrm{H}$ segregation. The atomic concentration of $\mathrm{H}$ atoms $\left(\rho_{\mathrm{H}}^{a}\right)$ in the model increases with the tensile strain, as shown in the right panel of Fig. 8(b). This phenomenon can be also observed from the volume density of the number of $\mathrm{H}$ atoms $\left(\rho_{\mathrm{H}}^{V}\right)$ in the slabs with the thickness of $5 \AA$ around the GB at various tensile strains, as shown in Fig. 8(c). The solubility of hydrogen in the twist model increases with the tensile strain (stress) in both GB and bulk zones. A crack embryo is nucleated at the strain of $12.2 \%$ that was introduced by the emission of dislocation with an excitation of the GB structure, as shown in Figs. 8(b) and 8(e). The crack embryo was gradually expanded until it crossed the entire interface at the strain of $12.5 \%$; i.e., the H-charging twist model was completely fractured. Then, $70 \%$ of the $\mathrm{H}$ atoms in the model were uncharged during the fracture process, as shown in the right panel in Fig. 8(b).

Defects of vacancy (Vac) and self-interstitial atom (SIA), created by the gliding of dislocation (Dis), were left in the separated grains, and the $\mathrm{H}$ atoms were mainly distributed 
in these defects and the newly created surfaces, as shown in the snapshot $(17.0 \%)$ in Fig. 8(e). There is an interesting phenomenon shown in the snapshot $(14.0 \%)$ in Fig. 8(e): the hydrogen concentration in the upper grain is significantly higher than that in the lower grain. The defect density (or the stress field introduced by the defects) might have a strong influence on hydrogen desorption.

Based on the above-mentioned two tensile tests, we observed that the presence of $\mathrm{H}$ atoms can facilitate dislocation emission from GB. The interaction between dislocation and $\mathrm{GB}$, including dislocation emission from GB and impingement on GB, plays an important role in the process of crack embryo nucleation [106]. The actual fracture should be a result of several aspects; in addition to the interaction of dislocation and GB, the vacancy diffusion or clustering and nanovoid growth should also be involved. However, the effects of vacancy and nanovoids were not observed owing to the high strain rate adopted in this study.

Note that in actual materials, many preexisting dislocations exist. Thus, these dislocations can move at a much lower stress level than the stress level observed in the above simulation starting from the preexisting dislocation-free condition. Some of the moving dislocations may impinge on the GB and excite the GB structure at a much lower stress level than that in our simulation, eventually leading to the nucleation of the crack embryo. However, the stress level of the nucleation of the crack embryo should be at a similar level as that in the above simulation because the nucleation of the crack embryo requires a bond breaking. Moreover, the local stress level at the tip of a propagating crack must be at the same level as that in our simulation; thus, similar phenomena can be observed at the tip of the crack.

\section{CONCLUSION}

A general-purpose NNIP for the $\alpha$-iron and hydrogen binary system was constructed based on the DFT reference database. This NNIP has been comprehensively tested for both trained and untrained data. The presented NNIP is reliable for the description of pure $\alpha$-iron and the interactions of hydrogen with bulk and various defects in $\alpha$-iron, including vacancies, surfaces, grain boundaries, and screw dislocation. Importantly, by virtue of the excellent flexibility of NNIP, this NNIP can solve several challenges facing Fe-H EAM potentials, such as the bond-breaking and bond-forming processes of $\mathrm{H}$ atoms at the iron surfaces and the energetics of screw dislocation motion.

The constructed NNIP was applied to several systems at finite temperature with the consideration of the HE process. The hydrogen charging and diffusion processes for $\Sigma 5(310)[001]$ tilt GB and $\frac{1}{2}[111]$ screw dislocation can be directly observed at the atomic level: the tilt GB is a ditch for hydrogen diffusion, while the screw dislocation is a low-efficiency pipe for hydrogen transportation. $\mathrm{H}$ atoms are trapped and distributed within a small range around the defects of vacancy, tilt GB, and screw dislocation. The desorption temperature of the trapped $\mathrm{H}$ atoms agrees with experimental observations. In comparison in MD-GCMC hybrid tensile tests for a twist GB with and without hydrogen charging, the presence of hydrogen can facilitate dislocation emission from the GB, eventually leading to nucleation of the crack embryo at the GB region. The growth of eth crack embryo results in intergranular fracture. This proved that the GB-dislocation reaction is an important piece of the HE process. These applications showed that the constructed NNIP has a potential contribution to the understanding of the physics in the HE process.

\section{ACKNOWLEDGMENTS}

F.-S.M. and S.O. acknowledge the support by JFE Steel Corporation, and the Elements Strategy Initiative for Structural Materials (ESISM). All calculations were performed on the large-scale computer systems at the Cybermedia Center of Osaka University and the Center for Computational Materials Science, IMR, Tohoku University (MASAMUNE-IMR).
[1] W. H. Johnson, On some remarkable changes produced in iron and steel by the action of hydrogen and acids, Nature (London) 11, 393 (1875).

[2] J. Hou, X.-S. Kong, X. Wu, J. Song, and C. Liu, Predictive model of hydrogen trapping and bubbling in nanovoids in bcc metals, Nat. Mater. 18, 833 (2019).

[3] P. Gong, J. Nutter, P. Rivera-Diaz-Del-Castillo, and W. Rainforth, Hydrogen embrittlement through the formation of low-energy dislocation nanostructures in nanoprecipitation-strengthened steels, Sci. Adv. 6, eabb6152 (2020).

[4] Y.-S. Chen, H. Lu, J. Liang, A. Rosenthal, H. Liu, G. Sneddon, I. McCarroll, Z. Zhao, W. Li, A. Guo et al., Observation of hydrogen trapping at dislocations, grain boundaries, and precipitates, Science 367, 171 (2020).

[5] M. Koyama, S. M. Taheri-Mousavi, H. Yan, J. Kim, B. C. Cameron, S. S. Moeini-Ardakani, J. Li, and C. C. Tasan, Origin of micrometer-scale dislocation motion during hydrogen desorption, Sci. Adv. 6, eaaz1187 (2020).
[6] G. P. Pun, R. Batra, R. Ramprasad, and Y. Mishin, Physically informed artificial neural networks for atomistic modeling of materials, Nat. Commun. 10, 2339 (2019).

[7] B.-J. Lee and J.-W. Jang, A modified embedded-atom method interatomic potential for the Fe-H system, Acta Mater. 55, 6779 (2007).

[8] M. Ruda, D. Farkas, and J. Abriata, Embedded-atom interatomic potentials for hydrogen in metals and intermetallic alloys, Phys. Rev. B 54, 9765 (1996).

[9] M. Wen, X.-J. Xu, S. Fukuyama, and K. Yokogawa, Embedded-atom-method functions for the body-centeredcubic iron and hydrogen, J. Mater. Res. 16, 3496 (2001).

[10] A. Ramasubramaniam, M. Itakura, and E. A. Carter, Interatomic potentials for hydrogen in $\alpha$-iron based on density functional theory, Phys. Rev. B 79, 174101 (2009).

[11] M. Mendelev, S. Han, D. Srolovitz, G. Ackland, D. Sun, and M. Asta, Development of new interatomic potentials appropriate for crystalline and liquid iron, Philos. Mag. 83, 3977 (2003). 
[12] G. Ackland, M. Mendelev, D. Srolovitz, S. Han, and A. Barashev, Development of an interatomic potential for phosphorus impurities in $\alpha$-iron, J. Phys.: Condens. Matter 16, S2629 (2004).

[13] J. Song and W. Curtin, Atomic mechanism and prediction of hydrogen embrittlement in iron, Nat. Mater. 12, 145 (2013).

[14] M. Wen, A new interatomic potential describing $\mathrm{Fe}-\mathrm{H}$ and $\mathrm{H}-$ $\mathrm{H}$ interactions in bcc iron, Comput. Mater. Sci. 197, 110640 (2021).

[15] M. Itakura, H. Kaburaki, and M. Yamaguchi, First-principles study on the mobility of screw dislocations in bcc iron, Acta Mater. 60, 3698 (2012).

[16] H. Mori and T. Ozaki, Neural network atomic potential to investigate the dislocation dynamics in bcc iron, Phys. Rev. Materials 4, 040601 (2020).

[17] X. Zhou, M. E. Foster, J. A. Ronevich, and C. W. San Marchi, Review and construction of interatomic potentials for molecular dynamics studies of hydrogen embrittlement in Fe-C based steels, J. Comput. Chem. 41, 1299 (2020).

[18] J. Behler and M. Parrinello, Generalized Neural-Network Representation of High-Dimensional Potential-Energy Surfaces, Phys. Rev. Lett. 98, 146401 (2007).

[19] A. P. Bartók, M. C. Payne, R. Kondor, and G. Csányi, Gaussian Approximation Potentials: The Accuracy of Quantum Mechanics, Without the Electrons, Phys. Rev. Lett. 104, 136403 (2010).

[20] M. Stricker, B. Yin, E. Mak, and W. Curtin, Machine learning for metallurgy II. A neural-network potential for magnesium, Phys. Rev. Materials 4, 103602 (2020).

[21] D. Marchand, A. Jain, A. Glensk, and W. Curtin, Machine learning for metallurgy I. A neural-network potential for Al$\mathrm{Cu}$, Phys. Rev. Materials 4, 103601 (2020).

[22] A. P. Bartók, J. Kermode, N. Bernstein, and G. Csányi, Machine Learning a General-Purpose Interatomic Potential for Silicon, Phys. Rev. X 8, 041048 (2018).

[23] C. W. Rosenbrock, K. Gubaev, A. V. Shapeev, L. B. Pártay, N. Bernstein, G. Csányi, and G. L. Hart, Machine-learned interatomic potentials for alloys and alloy phase diagrams, npj Comput. Mater. 7, 24 (2021).

[24] E. Davidson, T. Daff, G. Csanyi, and M. Finnis, Grand canonical approach to modeling hydrogen trapping at vacancies in $\alpha$-Fe, Phys. Rev. Materials 4, 063804 (2020).

[25] J. Behler, Constructing high-dimensional neural network potentials: A tutorial review, Int. J. Quantum Chem. 115, 1032 (2015).

[26] G. Sivaraman, A. N. Krishnamoorthy, M. Baur, C. Holm, M. Stan, G. Csányi, C. Benmore, and Á. Vázquez-Mayagoitia, Machine-learned interatomic potentials by active learning: Amorphous and liquid hafnium dioxide, npj Comput. Mater. 6, 104 (2020).

[27] See Supplemental Material at http://link.aps.org/ supplemental/10.1103/PhysRevMaterials.5.113606 for additional information.

[28] A. Pukrittayakamee, M. Malshe, M. Hagan, L. Raff, R. Narulkar, S. Bukkapatnum, and R. Komanduri, Simultaneous fitting of a potential-energy surface and its corresponding force fields using feedforward neural networks, J. Chem. Phys. 130, 134101 (2009).
[29] J. Behler, Atom-centered symmetry functions for constructing high-dimensional neural network potentials, J. Chem. Phys. 134, 074106 (2011).

[30] A. Singraber, T. Morawietz, J. Behler, and C. Dellago, Parallel multistream training of high-dimensional neural network potentials, J. Chem. Theory Comput. 15, 3075 (2019).

[31] S. Plimpton, Fast parallel algorithms for short-range molecular dynamics, J. Comput. Phys. 117, 1 (1995).

[32] A. Singraber, J. Behler, and C. Dellago, Library-based LAMMPS implementation of high-dimensional neural network potentials, J. Chem. Theory Comput. 15, 1827 (2019).

[33] https://github.com/mengfsou/NNIP-FeH.

[34] G. Imbalzano, A. Anelli, D. Giofré, S. Klees, J. Behler, and M. Ceriotti, Automatic selection of atomic fingerprints and reference configurations for machine-learning potentials, J. Chem. Phys. 148, 241730 (2018).

[35] G. Kresse and J. Furthmüller, Efficient iterative schemes for ab initio total-energy calculations using a plane-wave basis set, Phys. Rev. B 54, 11169 (1996).

[36] G. Kresse and D. Joubert, From ultrasoft pseudopotentials to the projector augmented-wave method, Phys. Rev. B 59, 1758 (1999).

[37] J. P. Perdew, K. Burke, and M. Ernzerhof, Generalized Gradient Approximation Made Simple, Phys. Rev. Lett. 77, 3865 (1996).

[38] H. Kimizuka and M. Shiga, Two distinct non-Arrhenius behaviors of hydrogen diffusivities in fcc aluminum, silver, and copper determined by ab initio path integral simulations, Phys. Rev. Materials 5, 065406 (2021).

[39] N. Artrith and J. Behler, High-dimensional neural network potentials for metal surfaces: A prototype study for copper, Phys. Rev. B 85, 045439 (2012).

[40] Z. S. Basinski, W. Hume-Rothery, and A. Sutton, The lattice expansion of iron, Proc. R. Soc. London A 229, 459 (1955).

[41] Y. S. Touloukian, R. Kirby, R. Taylor, and P. Desai, Thermal Expansion-Metallic Elements and Alloys, Thermophysical Properties of Matter Vol. 12 (IFI/Plenum, New York, 1975).

[42] X. Sha and R. Cohen, Lattice dynamics and thermodynamics of bcc iron under pressure: First-principles linear response study, Phys. Rev. B 73, 104303 (2006).

[43] T. Yokoyama and K. Eguchi, Anharmonicity and Quantum Effects in Thermal Expansion of an Invar Alloy, Phys. Rev. Lett. 107, 065901 (2011).

[44] J. J. Adams, D. Agosta, R. Leisure, and H. Ledbetter, Elastic constants of monocrystal iron from 3 to $500 \mathrm{~K}$, J. Appl. Phys. 100, 113530 (2006).

[45] J. Beeler, Jr. and R. Johnson, Vacancy clusters in $\alpha$-iron, Phys. Rev. 156, 677 (1967).

[46] D. Kandaskalov, C. Mijoule, and D. Connétable, Study of multivacancies in alpha Fe, J. Nucl. Mater. 441, 168 (2013).

[47] L. De Schepper, D. Segers, L. Dorikens-Vanpraet, M. Dorikens, G. Knuyt, L. Stals, and P. Moser, Positron annihilation on pure and carbon-doped $\alpha$-iron in thermal equilibrium, Phys. Rev. B 27, 5257 (1983).

[48] M.-C. Marinica, F. Willaime, and N. Mousseau, Energy landscape of small clusters of self-interstitial dumbbells in iron, Phys. Rev. B 83, 094119 (2011). 
[49] C.-C. Fu, F. Willaime, and P. Ordejón, Stability and Mobility of Mono- and Di-Interstitials in $\alpha$-Fe, Phys. Rev. Lett. 92, 175503 (2004).

[50] D. Dragoni, T. D. Daff, G. Csányi, and N. Marzari, Achieving DFT accuracy with a machine-learning interatomic potential: Thermomechanics and defects in bcc ferromagnetic iron, Phys. Rev. Materials 2, 013808 (2018).

[51] S. Klotz and M. Braden, Phonon Dispersion of bec Iron to 10 GPa, Phys. Rev. Lett. 85, 3209 (2000).

[52] J. Wang, G. K. Madsen, and R. Drautz, Grain boundaries in bcc-Fe: A density-functional theory and tightbinding study, Modell. Simul. Mater. Sci. Eng. 26, 025008 (2018).

[53] D. Scheiber, R. Pippan, P. Puschnig, and L. Romaner, Ab initio calculations of grain boundaries in bcc metals, Modell. Simul. Mater. Sci. Eng. 24, 035013 (2016).

[54] S. K. Bhattacharya, S. Tanaka, Y. Shiihara, and M. Kohyama, $\mathrm{Ab}$ initio perspective of the $\langle 110\rangle$ symmetrical tilt grain boundaries in bcc Fe: Application of local energy and local stress, J. Mater. Sci. 49, 3980 (2014).

[55] A. Togo and I. Tanaka, First principles phonon calculations in materials science, Scr. Mater. 108, 1 (2015).

[56] V. Vítek, Intrinsic stacking faults in body-centred cubic crystals, Philos. Mag. 18, 773 (1968).

[57] C. R. Weinberger, B. L. Boyce, and C. C. Battaile, Slip planes in bcc transition metals, Int. Mater. Rev. 58, 296 (2013).

[58] L. Proville, L. Ventelon, and D. Rodney, Prediction of the kink-pair formation enthalpy on screw dislocations in $\alpha$-iron by a line tension model parametrized on empirical potentials and first-principles calculations, Phys. Rev. B 87, 144106 (2013).

[59] J. Li, C.-Z. Wang, J.-P. Chang, W. Cai, V. V. Bulatov, K.-M. Ho, and S. Yip, Core energy and Peierls stress of a screw dislocation in bcc molybdenum: A periodic-cell tight-binding study, Phys. Rev. B 70, 104113 (2004).

[60] L. Dezerald, D. Rodney, E. Clouet, L. Ventelon, and F. Willaime, Plastic anisotropy and dislocation trajectory in BCC metals, Nat. Commun. 7, 11695 (2016).

[61] L. Dezerald, L. Proville, L. Ventelon, F. Willaime, and D. Rodney, First-principles prediction of kink-pair activation enthalpy on screw dislocations in bcc transition metals: $\mathrm{V}, \mathrm{Nb}$, Ta, Mo, W, and Fe, Phys. Rev. B 91, 094105 (2015).

[62] F. Maresca, D. Dragoni, G. Csányi, N. Marzari, and W. A. Curtin, Screw dislocation structure and mobility in body centered cubic Fe predicted by a Gaussian approximation potential, npj Comput. Mater. 4, 69 (2018).

[63] S. Shinzato, M. Wakeda, and S. Ogata, An atomistically informed kinetic Monte Carlo model for predicting solid solution strengthening of body-centered cubic alloys, Int. J. Plast. 122, 319 (2019).

[64] L. Dezerald, L. Ventelon, E. Clouet, C. Denoual, D. Rodney, and F. Willaime, $A b$ initio modeling of the two-dimensional energy landscape of screw dislocations in bec transition metals, Phys. Rev. B 89, 024104 (2014).

[65] L. Ventelon, F. Willaime, E. Clouet, and D. Rodney, Ab initio investigation of the Peierls potential of screw dislocations in bcc Fe and W, Acta Mater. 61, 3973 (2013).

[66] M. Wakeda, T. Tsuru, M. Kohyama, T. Ozaki, H. Sawada, M. Itakura, and S. Ogata, Chemical misfit origin of solute strengthening in iron alloys, Acta Mater. 131, 445 (2017).
[67] W. Geng, L. Wan, J.-P. Du, A. Ishii, N. Ishikawa, H. Kimizuka, and S. Ogata, Hydrogen bubble nucleation in $\alpha$-iron, Scr. Mater. 134, 105 (2017).

[68] P. Kuopanportti, E. Hayward, C.-C. Fu, A. Kuronen, and K. Nordlund, Interatomic Fe-H potential for irradiation and embrittlement simulations, Comput. Mater. Sci. 111, 525 (2016).

[69] W. Counts, C. Wolverton, and R. Gibala, First-principles energetics of hydrogen traps in $\alpha$-Fe: Point defects, Acta Mater. 58, 4730 (2010).

[70] Q. Chen, Q. Yao, Y.-L. Liu, Q.-F. Han, and F. Ding, Temperature-dependent dissolution and diffusion of $\mathrm{H}$ isotopes in iron for nuclear energy applications: First-principles and vibration spectrum predictions, Int. J. Hydrogen Energy 42, 11560 (2017).

[71] D. Jiang and E. A. Carter, Diffusion of interstitial hydrogen into and through bcc Fe from first principles, Phys. Rev. B 70, 064102 (2004).

[72] Y. He, Y. Li, C. Chen, and H. Yu, Diffusion coefficient of hydrogen interstitial atom in $\alpha$-Fe, $\gamma$-Fe and $\varepsilon$-Fe crystals by first-principle calculations, Int. J. Hydrogen Energy 42, 27438 (2017).

[73] M. Nagano, Y. Hayashi, N. Ohtani, M. Isshiki, and K. Igaki, Hydrogen diffusivity in high purity alpha iron, Scr. Metall. 16, 973 (1982).

[74] H. Kimizuka, H. Mori, and S. Ogata, Effect of temperature on fast hydrogen diffusion in iron: A path-integral quantum dynamics approach, Phys. Rev. B 83, 094110 (2011).

[75] T. Lu, G.-J. Niu, Y. Xu, J. Wang, Z. An, H. Liu, H. Zhou, F. Ding, G.-N. Luo, and X.-C. Li, Molecular dynamics study of the diffusion properties of $\mathrm{H}$ in $\mathrm{Fe}$ with point defects, Fusion Eng. Des. 113, 340 (2016)

[76] Y. Wang, D. Connétable, and D. Tanguy, Influence of trap connectivity on $\mathrm{H}$ diffusion: Vacancy trapping, Acta Mater. 103, 334 (2016).

[77] S. Li, Y. Li, Y.-C. Lo, T. Neeraj, R. Srinivasan, X. Ding, J. Sun, L. Qi, P. Gumbsch, and J. Li, The interaction of dislocations and hydrogen-vacancy complexes and its importance for deformation-induced proto nano-voids formation in $\alpha$-Fe, Int. J. Plast. 74, 175 (2015).

[78] Y. Tateyama and T. Ohno, Stability and clusterization of hydrogen-vacancy complexes in $\alpha$-Fe: An $a b$ initio study, Phys. Rev. B 67, 174105 (2003).

[79] E. Hayward, B. Beeler, and C. Deo, Multiple hydrogen trapping at monovacancies, Philos. Mag. Lett. 92, 217 (2012).

[80] K. Ohsawa, K. Eguchi, H. Watanabe, M. Yamaguchi, and M. Yagi, Configuration and binding energy of multiple hydrogen atoms trapped in monovacancy in bec transition metals, Phys. Rev. B 85, 094102 (2012).

[81] E. Hayward and C.-C. Fu, Interplay between hydrogen and vacancies in $\alpha$-Fe, Phys. Rev. B 87, 174103 (2013).

[82] K. Arakawa, A. Kageyama, H. Hiroshima, H. Yasuda, and S. Ogata, Hydrogen effects on the migration of nanoscale cavities in iron, ISIJ Int. 61, 2305 (2021).

[83] L. Xu, D. Kirvassilis, Y. Bai, and M. Mavrikakis, Atomic and molecular adsorption on Fe (110), Surf. Sci. 667, 54 (2018).

[84] W. Xie, L. Peng, D. Peng, F. L. Gu, and J. Liu, Processes of $\mathrm{H}_{2}$ adsorption on $\mathrm{Fe}(110)$ surface: A density functional theory study, Appl. Surf. Sci. 296, 47 (2014). 
[85] P. Chen, L. Qi, L. Zhang, W. Gao, and Q. Jiang, Adsorption behavior of gas molecules on hydrogenated Fe (111), Comput. Mater. Sci. 146, 261 (2018).

[86] A. Staykov, J. Yamabe, and B. P. Somerday, Effect of hydrogen gas impurities on the hydrogen dissociation on iron surface, Int. J. Quantum Chem. 114, 626 (2014).

[87] T. Wang, S. Wang, Q. Luo, Y.-W. Li, J. Wang, M. Beller, and H. Jiao, Hydrogen adsorption structures and energetics on iron surfaces at high coverage, J. Phys. Chem. C 118, 4181 (2014).

[88] F. Bozso, G. Ertl, M. Grunze, and M. Weiss, Chemisorption of hydrogen on iron surfaces, Appl. Surf. Sci. 1, 103 (1977).

[89] X. Zhou, N. Mousseau, and J. Song, Is Hydrogen Diffusion Along Grain Boundaries Fast or Slow? Atomistic Origin and Mechanistic Modeling, Phys. Rev. Lett. 122, 215501 (2019).

[90] S. Huang, D. Chen, J. Song, D. L. McDowell, and T. Zhu, Hydrogen embrittlement of grain boundaries in nickel: An atomistic study, npj Comput. Mater. 3, 28 (2017).

[91] Y. A. Du, J. Rogal, and R. Drautz, Diffusion of hydrogen within idealized grains of bcc Fe: A kinetic Monte Carlo study, Phys. Rev. B 86, 174110 (2012).

[92] S. Bechtle, M. Kumar, B. P. Somerday, M. E. Launey, and R. O. Ritchie, Grain-boundary engineering markedly reduces susceptibility to intergranular hydrogen embrittlement in metallic materials, Acta Mater. 57, 4148 (2009).

[93] A. Tahir, R. Janisch, and A. Hartmaier, Hydrogen embrittlement of a carbon segregated $\Sigma 5$ (310)[001] symmetrical tilt grain boundary in $\alpha$-Fe, Materi. Sci. Eng. A 612, 462 (2014).

[94] W. Geng, A. J. Freeman, R. Wu, C. Geller, and J. Raynolds, Embrittling and strengthening effects of hydrogen, boron, and phosphorus on a $\Sigma 5$ nickel grain boundary, Phys. Rev. B 60, 7149 (1999).

[95] M. Všianská and M. Šob, The effect of segregated spimpurities on grain-boundary and surface structure, magnetism and embrittlement in nickel, Prog. Mater. Sci. 56, 817 (2011).

[96] Y. A. Du, L. Ismer, J. Rogal, T. Hickel, J. Neugebauer, and R. Drautz, First-principles study on the interaction of $\mathrm{H}$ interstitials with grain boundaries in $\alpha$ - and $\gamma$-Fe, Phys. Rev. B 84, 144121 (2011)

[97] S. S. Kulkov, A. V. Bakulin, and S. E. Kulkova, Effect of boron on the hydrogen-induced grain boundary embrittlement in $\alpha$ Fe, Int. J. Hydrogen Energy 43, 1909 (2018).

[98] M. Itakura, H. Kaburaki, M. Yamaguchi, and T. Okita, The effect of hydrogen atoms on the screw dislocation mobility in bcc iron: A first-principles study, Acta Mater. 61, 6857 (2013).
[99] A. Ishii, J. Li, and S. Ogata, "conjugate channeling" effect in dislocation core diffusion: Carbon transport in dislocated bcc iron, PLoS One 8, e60586 (2013).

[100] Y. Zhao and G. Lu, Qm/mm study of dislocationhydrogen/helium interactions in $\alpha$-Fe, Modell. Simul. Mater. Sci. Eng. 19, 065004 (2011).

[101] J.-P. Du, W.-T. Geng, K. Arakawa, J. Li, and S. Ogata, Hydrogen-enhanced vacancy diffusion in metals, J. Phys. Chem. Lett. 11, 7015 (2020).

[102] W. Gerberich, R. Oriani, M.-J. Lji, X. Chen, and T. Foecke, The necessity of both plasticity and brittleness in the fracture thresholds of iron, Philos. Mag. A 63, 363 (1991).

[103] H. K. Birnbaum and P. Sofronis, Hydrogen-enhanced localized plasticity - a mechanism for hydrogen-related fracture, Mater. Sci. Eng. A 176, 191 (1994).

[104] M. Nagumo, Hydrogen related failure of steels—a new aspect, Mater. Sci. Technol. 20, 940 (2004).

[105] I. M. Robertson, P. Sofronis, A. Nagao, M. Martin, S. Wang, D. Gross, and K. Nygren, Hydrogen embrittlement understood, Metall. Mater. Trans. B 46, 1085 (2015).

[106] L. Wan, W. T. Geng, A. Ishii, J.-P. Du, Q. Mei, N. Ishikawa, H. Kimizuka, and S. Ogata, Hydrogen embrittlement controlled by reaction of dislocation with grain boundary in alpha-iron, Int. J. Plast. 112, 206 (2019).

[107] A. Stukowski, V. V. Bulatov, and A. Arsenlis, Automated identification and indexing of dislocations in crystal interfaces, Modell. Simul. Mater. Sci. Eng. 20, 085007 (2012).

[108] T. B. Flanagan, R. Balasubramaniam, and R. Kirchheim, Exploring lattice defects in palladium and its alloys using dissolved hydrogen, Platinum Met. Rev. 45, 114 (2001).

[109] D. Frenkel and B. Smit, Understanding Molecular Simulation: From Algorithms to Applications, Vol. 1 (Elsevier, Amsterdam, 2001).

[110] M. Iwamoto and Y. Fukai, Superabundant vacancy formation in iron under high hydrogen pressures: Thermal desorption spectroscopy, Mater. Trans. JIM 40, 606 (1999).

[111] K. Ono and M. Meshii, Hydrogen detrapping from grain boundaries and dislocations in high purity iron, Acta Metall. Mater. 40, 1357 (1992).

[112] O. Barrera, D. Bombac, Y. Chen, T. Daff, E. Galindo-Nava, P. Gong, D. Haley, R. Horton, I. Katzarov, J. Kermode et al., Understanding and mitigating hydrogen embrittlement of steels: A review of experimental, modelling and design progress from atomistic to continuum, J. Mater. Sci. 53, 6251 (2018).

[113] A. Stukowski, Computational analysis methods in atomistic modeling of crystals, JOM 66, 399 (2014). 